\title{
Fréedericksz-Like Transition in a Biaxial Smectic- $A$ Phase
}

\author{
Claire Meyer®, ${ }^{1, *}$ Patrick Davidson $\odot,{ }^{2}$ Doru Constantin, ${ }^{2}$ Vassili Sergan, ${ }^{3}$ Daniel Stoenescu $\odot,{ }^{4}$ \\ Anamarija Kneževićc®, ${ }^{5}$ Irena Dokli@ ${ }^{5}$ Andreja Lesac ${ }^{5},{ }^{5}$ and Ivan Dozov ${ }^{1,2}$ \\ ${ }^{1}$ Physique des Systèmes Complexes, Université de Picardie Jules Verne, 80039 Amiens, France \\ ${ }^{2}$ Laboratoire de Physique des Solides, Université Paris-Saclay, CNRS, 91405 Orsay, France \\ ${ }^{3}$ California State University, Sacramento, 6000 J Street, Sacramento, California 95819-6041, USA \\ ${ }^{4}$ Optics Department, IMT Atlantique, CS 83818, 29238 Brest cedex 3, France \\ ${ }^{5}$ Ruđer Bošković Institute, Bijenička 54, 10000 Zagreb, Croatia
}

(Received 1 March 2021; revised 11 May 2021; accepted 2 June 2021; published 16 July 2021)

\begin{abstract}
The two main classes of liquid-crystal (LC) phases of rodlike molecules are nematics, where the rods align in the same direction (the nematic director $\mathbf{n}$ ), and smectics, where the rods not only are aligned but also form layers. The electro-optic effects in LC devices that are a backbone in today's display industry mainly use the Fréedericksz transition, which is the bulk reorientation of a surface-anchored nematic by an electric field. Conventional (uniaxial) smectics do not present a Fréedericksz transition, because, due to their layered structure, the director reorientation would distort the layers, which would cost too much energy. In a worldwide ongoing effort to extend the variety of LC compounds suitable for applications in the display industry, bent-shaped molecules have recently raised much attention, since they present multiple new LC phases with unusual properties. In this paper, we report on a structural and electro-optic study of the LC phases of a bent-shaped dimer. On cooling from the isotropic liquid, this compound shows a usual nematic $(N)$, a twist-bend nematic $\left(N_{\mathrm{TB}}\right)$, and a biaxial smectic- $A$ phase $\left(\operatorname{Sm} A_{b}\right)$. Quite surprisingly, contrary to usual smectics, $\mathrm{Sm} A_{b}$ presents a remarkable electro-optic response, with low $(<4 \mathrm{~V})$ voltage threshold, no reorganization of the smectic layers, and low $(<1 \mathrm{~ms})$ response time (i.e., 30 times faster than the $N$ phase at higher temperature). We interpret this unexpected electro-optic effect as a Fréedericksz transition affecting the secondary director $\mathbf{m}$ of the $\operatorname{Sm} A_{b}$, and we model it by analogy with the usual Fréedericksz transition of the $\mathbf{n}$ director of the uniaxial $N$ phase. Indeed, a Fréedericksz transition affecting only $\mathbf{m}$ in this biaxial fluid smectic does not alter its layered structure and costs little energy. From the point of view of applications, thanks to its low relaxation time, this "biaxial" Fréedericksz transition could be exploited in electro-optic devices that require fast switching.
\end{abstract}

DOI: 10.1103/PhysRevX.11.031012

Subject Areas: Condensed Matter Physics, Soft Matter

\section{INTRODUCTION}

Liquid crystals (LCs) are intermediate phases (mesophases) observed, upon melting, between the crystalline state and the usual (isotropic) liquid state. By definition, LC phases are both fluid and anisotropic. The vast majority of LC compounds known today are rodlike organic molecules, and they offer two main types of mesophases called nematics and smectics. Nematic phases are the most disordered ones, as they have only long-range orientational order of the rodlike molecules; i.e., the molecules tend to

\footnotetext{
* Corresponding author. claire.meyer@u-picardie.fr

Published by the American Physical Society under the terms of the Creative Commons Attribution 4.0 International license. Further distribution of this work must maintain attribution to the author(s) and the published article's title, journal citation, and DOI.
}

align along a common direction called the director, $\mathbf{n}$. The most common nematic $(N)$ phase used in electro-optic displays has uniaxial symmetry around $\mathbf{n}$, but there are other kinds of nematics, such as chiral ones (the cholesteric and blue phases), the still elusive biaxial nematic, which has lower orientational symmetry, and the spontaneously modulated nematics where the orientational order is periodic in space. Smectics have lower symmetry than usual nematics, because they have, in addition to orientational order, long-range positional order of the centers of mass of the LC molecules in at least one direction of space, resulting in a layered structure. The most common smectic, called smectic $A(\operatorname{Sm} A)$, is a uniaxial phase where the director is parallel to the normal to the layers and the molecules have no long-range positional order within the layers, which are, therefore, fluid. In other smectic phases, the molecules may be tilted with respect to the normal to the layers $(\mathrm{SmC})$, or they may have long-range positional order within the layers $(\mathrm{Sm} B)$ or even both $(\mathrm{Sm} G)$. 
The Fréedericksz transition (FrTr) is arguably the most important electro-optic feature of nematic liquid crystals, as it is a basis of their widespread applications in display technology. This transition, discovered long ago [1], involves reorientation of the nematic optic axis (the director) by an applied electric field above a threshold value. It results from the competition of the electric torque acting on the bulk anisotropic material with the surface torque induced, at the sample boundaries, by the anchoring of $\mathbf{n}$ in a direction perpendicular to that imposed by the field. A similar FrTr has not yet been reported in smectics, because the twist and bend deformation of the director is topologically forbidden due to their layered structure. Instead, a small electro-optic effect, called a "ghost transition" [2,3], can barely be observed and is of no practical use for applications. Therefore, the FrTr is usually regarded as a distinctive property of the nematic LC phase.

The search for new LC compounds for electro-optic applications, in the course of the past 20 years, has led to the development of bent-shaped, bananalike, molecules that present a rich variety of LC phases, including many different new types of smectics showing spontaneous symmetry breaking and ferroelectricity [4-15]. Among these compounds, bent-shaped "dimer" molecules have recently gained particular interest worldwide, because they often display new types of nematic phases, namely, the "twist-bend" nematic [16-26] $\left(N_{\mathrm{TB}}\right)$ and the still elusive "splay-bend" nematic $\left(N_{\mathrm{SB}}[17,24,27-31]\right)$. In addition to these modulated nematics, bent-shaped dimer molecules also show very original smectic phases with structures characterized by the intercalation of the monomer moieties of the molecules [7,9-13,32-42].

In this context, a bent-shaped dimer, 1,7-bis[6-(4-hexyloxybenzoyloxy)naphthalene-2-yl]heptane (labeled BNA76), is reported [26] to present a very peculiar LC phase, called $M_{X}$, whose structure and properties could not be determined because it is monotropic and easily crystallizes, thus precluding any electro-optic and structural investigations. In this work, using mixtures of BNA-76 with a small fraction of a rodlike nematic compound as a means to hinder crystallization, we show that the $M_{X}$ phase is actually a biaxial $\operatorname{Sm} A\left(\operatorname{Sm} A_{b}\right)$ comprised of completely intercalated layers. Moreover, quite surprisingly, this biaxial smectic phase shows a nematiclike electro-optic effect, with rather fast $(<1 \mathrm{~ms})$ relaxation times and low (approximately $4 \mathrm{~V}$ ) field threshold values. We interpret and model these observations as the signature of a FrTr of the secondary director $\mathbf{m}$ that defines the direction of the width of the lathlike molecule. Indeed, a transition affecting only the secondary director of a biaxial $\operatorname{Sm} A$ phase, called "biaxial Fréedericksz transition" (BFrTr) hereafter, can take place, since it does not distort the layered structure.

\section{EXPERIMENTAL RESULTS}

As many other bent-shaped dimers, BNA-76 (Fig. 1) shows a transition to the $N_{\mathrm{TB}}$ phase on cooling from the

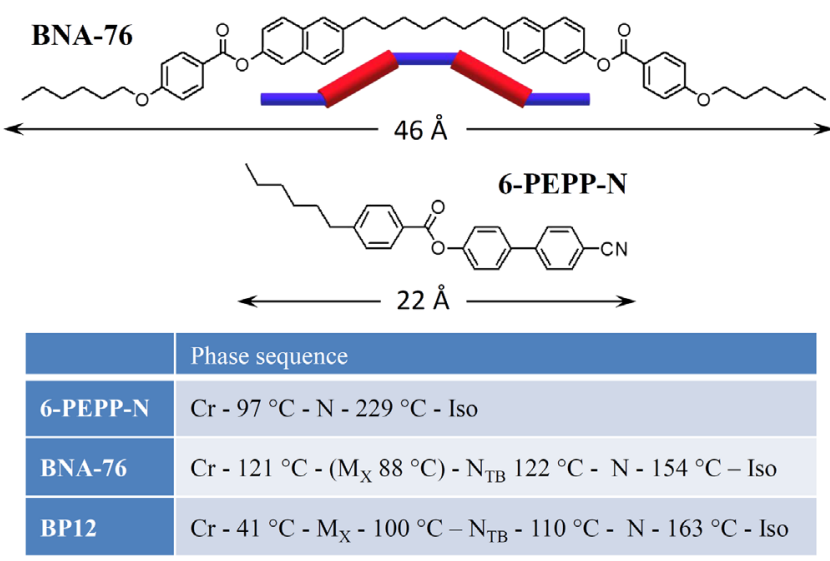

FIG. 1. Molecular structure and phase sequence of the LC compounds. For the BNA-76 bent-shaped dimer, a simplified sketch of the average conformation of the molecule is also shown, with the electron-rich conjugated parts in red and the alkyl and alkoxy chains in blue.

$N$ phase [26]. On further cooling, when the $N_{\text {Tв }}$ phase is supercooled by about $40^{\circ} \mathrm{C}$, another transition takes place, to a mesophase, labeled $M_{X}$, whose structure remains so far unknown. Its optical textures indicate that it is either a smectic or a modulated nematic $\left(N_{\mathrm{TB}}\right.$ or $\left.N_{\mathrm{SB}}\right)$ phase. However, because the $M_{X}$ phase is strongly supercooled, it crystallizes very fast in bulk samples, preventing its identification by x-ray scattering. To avoid crystallization, we prepare mixtures of BNA-76 with the rod-shaped nematogen 4'-cyano[1, 1'-biphenyl]-4-yl 4-hexylbenzoate (6-PEPP- $N$ ). This compound is selected for its high $N-I$ transition temperature, good miscibility with BNA-76, high dielectric anisotropy, and molecular size similar to that of the BNA-76 "monomer" (Fig. 1). Here, we focus on the "BP12" mixture (with $12 \mathrm{wt} \%$ of 6-PEPP- $N$ ) whose phase sequence (Fig. 1) is similar to that of pure BNA-76 but with an $N_{\mathrm{TB}}-M_{X}$ transition temperature higher by $30^{\circ} \mathrm{C}$ and, most importantly, a much lower crystallization temperature (see the Appendix A). Therefore, the $M_{X}$ phase of BP12 is thermodynamically stable at temperatures between $80^{\circ} \mathrm{C}$ and $100^{\circ} \mathrm{C}$, thus allowing for the $\mathrm{x}$-ray and electro-optic investigation of its structure and properties.

\section{A. Structural study}

X-ray scattering patterns (Fig. 2) of a BP12 sample in the $M_{X}$ phase, with the director aligned by a magnetic field, show two sharp reflections at small scattering angles, at $q_{0}=0.28 \AA^{-1}$. These resolution-limited reflections reveal the existence of a layered structure, i.e., a smectic phase. As expected, these reflections are replaced by wider and weaker diffuse scattering spots in the $N$ and $N_{\text {TB }}$ phases (see Appendix B, Fig. 15). The smectic period of the $M_{X}$ phase, $d_{0}=2 \pi / q_{0}=22.5 \AA$, is approximately half the length $(L=46 \AA)$ of the conformation of the BNA76 molecule estimated by the HyperChem package using 

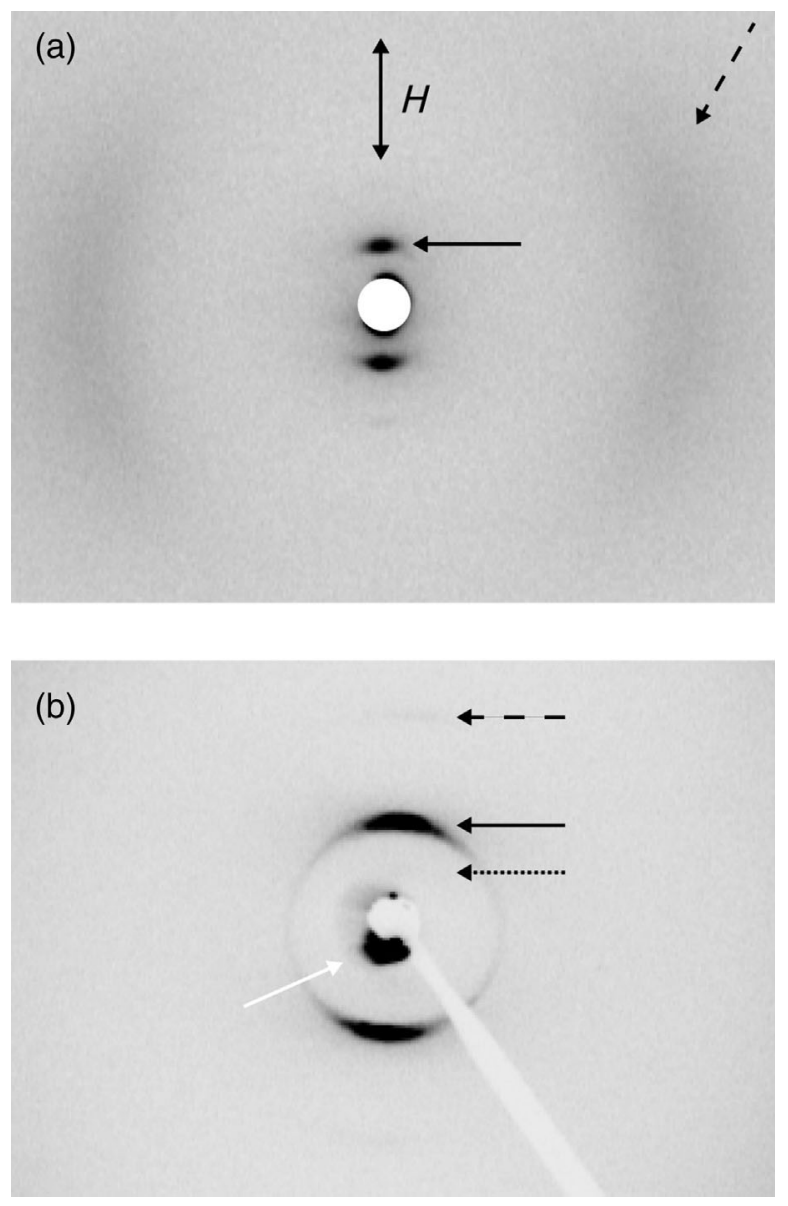

FIG. 2. X-ray scattering patterns of a BP12 sample in the $M_{X}$ phase $\left(T=97^{\circ} \mathrm{C}\right)$ aligned by a $1.7 \mathrm{~T}$ magnetic field $H$ (doubleheaded arrow). (a) With a $60 \mathrm{~mm}$ sample-to-detection distance. The solid arrow points to one of the smectic reflections at small angles, whereas the dashed arrow points at the wide-angle diffuse ring. (The white disk at the center represents the beam stop.) (b) With a $120 \mathrm{~mm}$ sample-to-detection distance. The solid arrow points to one of the smectic reflections, the dashed arrow to the barely observable second-order smectic reflection, and the dotted arrow to the absence of any scattering at wave vector $q_{0}=2 \pi / L$, where $L$ is the BNA-76 molecular length. (The white arrow points to parasitic scattering around the beam stop.)

the AM1 Hamiltonian [43]. No scattering is detected around $q_{0} / 2=0.14 \AA^{-1}$, which would correspond to the whole length of the molecule, and, therefore, the dimers are completely intercalated. Higher-resolution measurements of unaligned samples confirmed this conclusion (see Appendix B, Fig. 16).

In addition, a diffuse scattering ring, observed at wide angles, reveals the liquidlike character of the smectic layers. The maximum of this ring lies in the direction perpendicular to that of the smectic modulation, which suggests that the director is parallel to the normal to the layers, as in a smectic- $A(\operatorname{Sm} A)$ phase. However, the angular extension of the ring is very large, and, moreover,

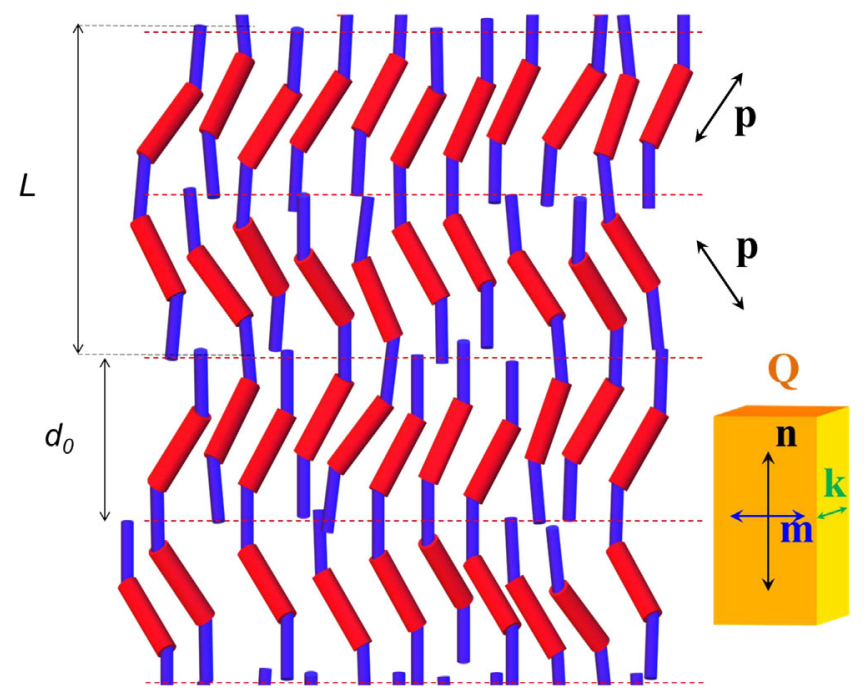

FIG. 3. Sketch of the structure of the $M_{X}$ phase. The monomer units form smectic liquid layers with thickness $d_{0} \approx L / 2$. In each layer, the main axes $\mathbf{p}$ of the monomers are tilted in the same direction, and the sign of the tilt alternates from one layer to the next. The dimers span two adjacent layers and form an orthogonal intercalated smectic phase. The orientational order tensor $\mathbf{Q}$ of the dimers (shown in orange) is biaxial, with primary director $\mathbf{n}$ oriented along the layers normal and secondary director $\mathbf{m}$ parallel to the projection of $\mathbf{p}$ on the layer plane. ( $\mathbf{k}$ is the third director, which is perpendicular to both $\mathbf{n}$ and $\mathbf{m}$.)

it increases with decreasing temperature (see Appendix B, Fig. 17). This last feature is quite unexpected for a common $\operatorname{Sm} A$ phase and suggests a tilt of the mesogenic cores in the layers, like in $\mathrm{SmC}$ or smectic $C_{A}\left(\mathrm{Sm}_{A}\right)$ phases.

Nevertheless, because $d_{0}=L / 2$, the long axis of the dimer is perpendicular to the layers, as confirmed by optical microscopy observations of the mutual orientation of the optic axis and the layers (see below). Consequently, considering the dimer molecules, the phase is smectic $A$, with $\mathbf{n}$ parallel to $\mathbf{q}_{0}$. However, the x-ray scattering results clearly indicate that the $M_{X}$ phase is an intercalated smectic, with the monomers tilted with respect to the normal to the layers, as in the intercalated phases already reported for other bent-shaped dimers [7,9-13,32-36,3842]. There are several structures, differing by the azimuthal correlations of the monomers, which are consistent with the $\mathrm{x}$-ray data (see Appendix F), but the one that agrees best with our whole set of results (see the next sections) is illustrated in Fig. 3. The layer thickness (i.e., the period of the mass density and electron density waves) is $d_{0} \approx L / 2$, meaning that the repeat unit of the smectic structure is not the dimer molecule but its mesogenic subunit (or "monomer"). Therefore, each dimer molecule must span two adjacent monomer layers. This intercalated structure is similar to that proposed before for the intercalated smectic phase of several bent-shaped dimers [9-11, 13,33,34,41,44-49]. The monomers have their main axes p tilted in the same way in each layer, but the tilt direction 
alternates from one layer to the next, as in an anticlinic smectic $C\left(\mathrm{Sm}_{A}\right) \quad[4,42,49,50]$ (see Sec. III and Appendix F).

\section{B. Optical and electro-optical study}

\section{Texture observations with or without a field}

For optical and electro-optical experiments, we use sandwich cells with substrates treated to provide planar alignment (see Appendix A for details). The cell is filled by capillarity with the LC mixture in the isotropic phase and then cooled to the nematic phase, resulting in uniform planar alignment of the nematic director $\mathbf{n}$ parallel to the rubbing direction $\mathbf{r}$. Slowly cooling the sample across the $N-N_{\mathrm{TB}}$ sharp transition, we observe at $T=108.9^{\circ} \mathrm{C}$ the growth of large monochiral $N_{\mathrm{TB}}$ domains with optical axis $\mathbf{N}$, also parallel to $\mathbf{r}$, and alternating handedness of the chirality (Figs. 18 and 19). Upon further cooling, to avoid the typical stripe instabilities of the $N_{\mathrm{TB}}$ phase [see Fig. 20(a)], the sample is annealed using temperature oscillations and simultaneous voltage bursts (see Appendix $\mathrm{C}$ for details).

The $N_{\mathrm{TB}}-M_{X}$ phase transition starts at $T \approx 103.5^{\circ} \mathrm{C}$ and, contrary to the sharp $N-N_{\text {TB }}$ transition, has a very large (about $5^{\circ} \mathrm{C}$ ) temperature range of biphasic coexistence. Starting from a well-annealed $N_{\text {TB }}$ texture and slowly decreasing the temperature (approximately $0.1^{\circ} \mathrm{C} / \mathrm{min}$ ), we are able to grow highly uniform single domains of the $M_{X}$ phase [Fig. 4(a)]. These domains are birefringent, and their slow (higher-index) axis $\mathbf{N}$ has the same orientation as that in the surrounding $N_{\mathrm{TB}}$ regions, $\mathbf{N} \| \mathbf{r}$. However, they can be easily recognized in polarized light [Figs. 4(a) and 4(b)], because their birefringence is larger
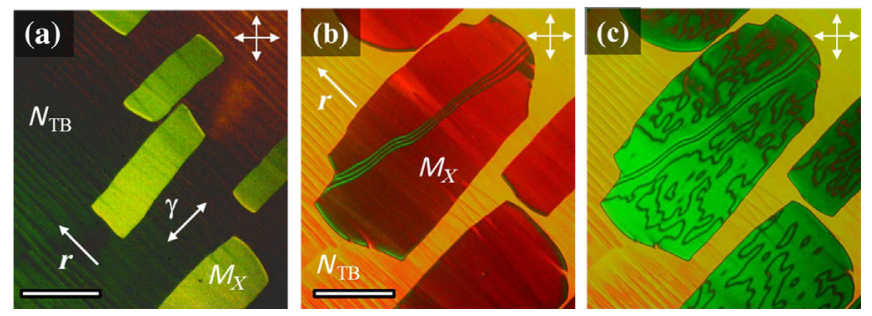

FIG. 4. Coexistence of uniform single domains of the $M_{X}$ and $N_{\text {TB }}$ phases of the BP12 mixture (planar cell, $d=9.8 \mu \mathrm{m}$, scale bar $100 \mu \mathrm{m}$ ). The optic axes of both phases are approximately parallel to the rubbing direction $\mathbf{r}$. The cell is viewed between crossed polarizers, using a Berek compensator with slow axis $\gamma \perp$ r. (a) Growth of uniform $M_{X}$ domains within the $N_{\mathrm{TB}}$ at $T=102.4{ }^{\circ} \mathrm{C}$. (b),(c) $M_{X}$ and $N_{\mathrm{TB}}$ domains at $T=100.7^{\circ} \mathrm{C}$, without a field (b) and when a biaxial Fréedericksz transition (c) is induced by an ac field $\left(U_{\mathrm{rms}}=9 \mathrm{~V}, f=6 \mathrm{kHz}\right)$ applied along the cell normal. The birefringence of the $N_{\mathrm{TB}}$ [yellow regions in (b) and (c)] is independent of the field. The birefringence of the $M_{X}$ phase varies with the field, from its low zerofield value [brown-red domains in (b)] to a high value above the transition [green domains in (c)]. The defect walls that separate the twin domains in (c) appear as thick dark lines. than that of the $N_{\mathrm{TB}}$ phase. In the biphasic temperature range, the $M_{X}$ single domains progressively grow as the temperature is slowly decreased, until the whole sample turns into the $M_{X}$ phase. At constant temperature, the coexisting $M_{X}$ and $N_{\mathrm{TB}}$ regions are at equilibrium (Fig. 21), and the proportions of the two phases depend only on the temperature, in a reversible way. The analysis of the textures shows that the primary director $\mathbf{n}$ of the $M_{X}$ phase is parallel to the helix axis $\mathbf{h}$ of the $N_{\mathrm{TB}}$ phase and that the smectic layers of the $M_{X}$ domains remain parallel to the $N_{\text {TB }}$ pseudolayers (and perpendicular to $\mathbf{r}$ ).

Surprisingly enough for a smectic phase, the birefringence of the $M_{X}$ domains changes when an electric field is applied to the cell [Figs. 4(b) and 4(c)]. Several features of this electro-optic effect are reminiscent of the classical FrTr in the $N$ phase (i.e., the transition observed in exactly the same cell above the $N_{\mathrm{TB}}-N$ transition temperature). The transition has a well-defined voltage threshold $U_{c} \sim 4 \mathrm{~V}$ rms, which is independent of the cell thickness. $U_{c}$ is rather low and is not much higher than the FrTr threshold (approximately $2 \mathrm{~V} \mathrm{rms)} \mathrm{in} \mathrm{the} \mathrm{nematic} \mathrm{phase.} \mathrm{Apart} \mathrm{from}$ the variation in birefringence, there is no texture change at $U>U_{c}$, which clearly shows that there is no reorientation of the smectic layers at the transition. This result is confirmed by the reversibility and absence of hysteresis of the effect upon field removal. Finally, the orientation of the slow axis of the $M_{X}$ domain does not change when the field is applied [Figs. 22(b) and 22(d)], which is also similar to the nematic FrTr.

However, the voltage dependence of the birefringence is strikingly different in the $M_{X}$ phase because the birefringence increases with $U$ [for quantitative measurements, see Fig. 6(a) in the next section], whereas it decreases toward zero with increasing voltage for the nematic FrTr. Therefore, qualitatively, this behavior proves that, unlike the nematic case, the electro-optic transition in the $M_{X}$ phase is not related to a rotation of the primary director $\mathbf{n}$ but is instead due to a rotation of the order-parameter tensor $\mathbf{Q}$ around $\mathbf{n}$. In other words, this behavior is a clear sign that the $M_{X}$ phase is biaxial and that the birefringence variation is related only to an out-of-plane rotation of the secondary macroscopic director $\mathbf{m}$.

To study the field-induced transition in the $M_{X}$ phase in more detail, the cell is observed between crossed polarizers, with a wideband interferential filter centered at $\lambda=546 \mathrm{~nm}$ and a Berek compensator adjusted to give optimal contrast between the different coexisting phases and textures [Figs. 4(b) and 4(c)]. In these conditions, the $N_{\text {Тв }}$ phase appears yellow, the low-birefringence (LB) $M_{X}$ state, with in-plane orientation of both $\mathbf{n}$ and $\mathbf{m}$, appears brown-red, and the high-birefringence (HB) $M_{X}$ state, with out-ofplane tilt of $\mathbf{m}$, appears green. Without the field [Fig. 4(b)], the coexisting $N_{\mathrm{TB}}$ and $M_{X}$ regions are uniform, both with the slow axis parallel to $\mathbf{r}$ but with slightly different birefringence. The $M_{X}$ region is mainly in the LB state, 
except for occasional defects that are walls with $\pi$ reorientation of $\mathbf{m}$ on the boundary surfaces. The $\pi$ walls separate two domains with antiparallel surface anchoring of $\mathbf{m}$. This doubly degenerate orientation of $\mathbf{m}$, which is parallel to the surface and perpendicular to $\mathbf{r}$, corresponds to anchoring-energy minima. The color in the middle of the $\pi$ wall is green, because $\mathbf{m}$ is perpendicular to the surface (in the whole bulk of the wall) and the birefringence is higher, as in the HB state observed under the field.

When the field is applied [Fig. 4(c)], the $N_{\mathrm{TB}}$ regions remain unchanged, but the $M_{X}$ domains undergo a transition to the HB state which is recognized by its green color. In the HB state, in the middle of the cell, $\mathbf{m}$ is tilted away from the surface plane. Because of the double degeneracy of the tilt, there appear two topologically different HB states with the same energy. This situation is similar to the twin domains observed above the FrTr in nematic samples with no pretilt [51]. In the walls between the domains, the tilt angle of $\mathbf{m}$ decreases to zero in the middle of the wall and then increases again with the opposite sign. Therefore, the birefringence in the wall is lower (in the middle, it is the same as in the LB state), and the walls are easily distinguished in the images as thick darker lines separating the twin domains. When the field is suddenly applied, the fast reorientation process creates twin domains. Then, due to the higher energy of the walls, the smaller twin domains progressively shrink and disappear, and the texture of the $M_{X}$ region coarsens (see Supplemental Material [52] for video of the coarsening process). At the end of this coarsening process, only a few twin domains remain in the $M_{X}$ region. They are stabilized by the occasional pinning of the walls on the cell surfaces and the $N_{\mathrm{TB}}-M_{X}$ interface.

The most direct way to prove the biaxiality of the $M_{X}$ phase consists in observing and measuring a finite birefringence in a homeotropically aligned sample (i.e., with $\mathbf{n}$ parallel to the cell normal and to the observation direction). Despite the notorious difficulty of producing a surfaceinduced homeotropic alignment of bent-shaped dimers, we achieve a quasihomeotropic alignment in our cells by applying a strong field $(E=17 \mathrm{~V} / \mu \mathrm{m}$; see Appendix C for details). Figure 5 shows the growth under a field of large and uniform quasihomeotropic $M_{X}$ domains within a quasihomeotropic $N_{\mathrm{TB}}$ region. These domains are indeed birefringent, and their slow axis is parallel to $\mathbf{r}$. Their birefringence $(\Delta n \approx 0.02)$ is much smaller than that of a planar $M_{X}$ domain $(\Delta n \approx 0.14)$ but is significantly larger than that of the adjacent quasihomeotropic $N_{\text {TB }}$ region. The birefringence is independent of the temperature and the field strength (as long as it remains strong enough to maintain the quasihomeotropic texture). During the growth of the $M_{X}$ domains under a strong field, we do not observe any texture change or instability, which suggests that $\mathbf{n}$ in the $M_{X}$ phase is parallel to the helix axis of the quasihomeotropic $N_{\mathrm{TB}}$ phase surrounding it. Then, the $M_{X}$ smectic layers are parallel to the cell surfaces and keep the
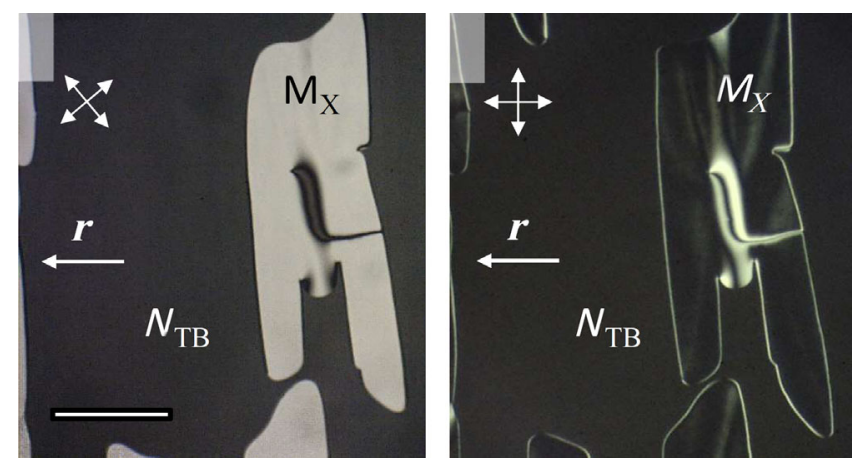

FIG. 5. Growth of a homeotropic $M_{X}$ domain within the $N_{\mathrm{TB}}$ under an electric field $\left(U_{\mathrm{rms}}=25 \mathrm{~V}, d=1.4 \mu \mathrm{m}, T=100{ }^{\circ} \mathrm{C}\right)$. The observation of birefringence while rotating the crossed polarizers (white double-headed arrows) reveals that the domain is biaxial, with m parallel to $\mathbf{r}$. (Scale bar $100 \mu \mathrm{m}$.)

orientation of the $N_{\text {TB }}$ pseudolayers. The rotation of the cell between crossed polarizers reveals (Fig. 5) that $\mathbf{m}$ is parallel to the cell surface (and the smectic layers) and to the rubbing direction in the bulk of the $M_{X}$ phase. To conclude on this point, the observation of birefringence while examining the cell along $\mathbf{n}$ is a direct proof of the lack of revolution symmetry around $\mathbf{n}$, i.e., that the $M_{X}$ phase is biaxial. This conclusion is further confirmed by additional analyses of texture instabilities (see Appendix C).

\section{Birefringence measurements}

These qualitative proofs of the biaxiality of the $M_{X}$ phase are quantitatively confirmed by the measurement (see Appendix A) of the birefringence with or without applied fields. The temperature dependence of the birefringence measured in all the phases of the BP12 mixture is shown in Fig. 6(a). For $T>140{ }^{\circ} \mathrm{C}$, the birefringence has the usual behavior expected for a nematic phase. Upon decreasing the temperature, it increases rapidly and follows the Haller law [53] $\Delta n=\Delta n_{0}\left(1-T / T^{*}\right)^{\beta}$, with $\Delta n_{0}=0.253$, $\beta=0.20$, and $T^{*}=160.9^{\circ} \mathrm{C}$, corresponding to the $N-I$ transition temperature. However, below $140{ }^{\circ} \mathrm{C}$, still in the nematic phase, the values of $\Delta n$ are significantly smaller than those extrapolated from the Haller-law fit [red line in Fig. 6(a)]. Such deviation is typical for the bent-shaped dimers that present the $N_{\mathrm{TB}}$ phase [31,54-57]. $\Delta n$ decreases further in the $N_{\mathrm{TB}}$, which is due to the temperature variation of the heliconical tilt angle, as already reported for other $N_{\mathrm{TB}}$-forming compounds [31,54,55,57].

When the sample is cooled into the $M_{X}-N_{\mathrm{TB}}$ coexistence range, the birefringence of the $N_{\mathrm{TB}}$ regions remains unchanged, as expected. Without the field, the birefringence of the $M_{X}$ domains, $\Delta n=n_{n n}-n_{m m}$, is significantly higher than that in the $N_{\text {TВ }}$ phase, $\Delta n=n_{\|}-n_{\perp}$. It is closer to the extrapolated Haller fit, but it also remains practically constant throughout the whole temperature range of coexistence (the weak slope of the curves is an 

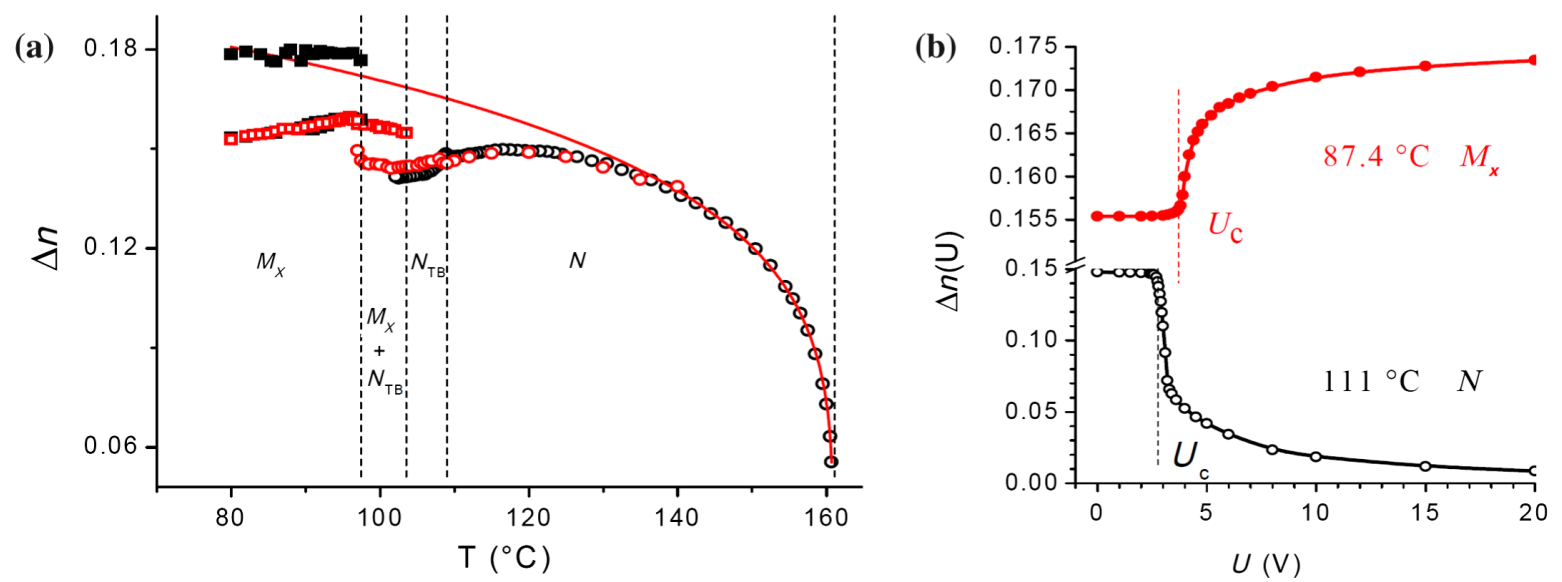

FIG. 6. Temperature and field dependence of the birefringence of the BP12 mixture. (a) $\Delta n(T)$ measured in different phases and for different sample alignments. The black symbols show the data obtained using PMT detection, whereas the red symbols show the data measured with the image-mapping technique. The circles show the data $\Delta n=n_{\|}-n_{\perp}$, obtained in the uniaxial $N$ and $N_{\mathrm{TB}}$ phases or regions, whereas the open squares show the data $\Delta n=n_{n n}-n_{m m}$, obtained in the $M_{X}$ phase without a field. The full squares show the data $\Delta n=n_{n n}-n_{k k}$ in the $M_{X}$ phase, obtained by extrapolation of the results measured under a field. The red line shows the birefringence expected by extrapolation of the Haller fit [53] of the data in the range $140{ }^{\circ} \mathrm{C}-160{ }^{\circ} \mathrm{C}$. (b) $\Delta n(U)$ in the $N$ (black symbols) and $M_{X}$ (red symbols) phases.

artifact due mainly to light scattering by the heterogeneous biphasic texture). On further cooling, when the last $N_{\text {TB }}$ domains disappear, the birefringence of the $M_{X}$ phase decreases slowly, indicating a weak temperature dependence of its biaxial order-parameter tensor.

Under the field, in the $N$ and $N_{\text {TB }}$ phases, the only possible reorientation is the out-of-plane rotation of the optic axis $\mathbf{N}$, which decreases the birefringence measured at normal light incidence [Fig. 6(b), black symbols]. The field effect is more subtle in the biaxial $M_{X}$ phase. When cooling the sample from the $N$ to the $M_{X}$ phase under a strong field, the $M_{X}$ domains grow with $\mathbf{n}$ parallel to the field (see Fig. 5) and the smectic layers parallel to the surface, and the

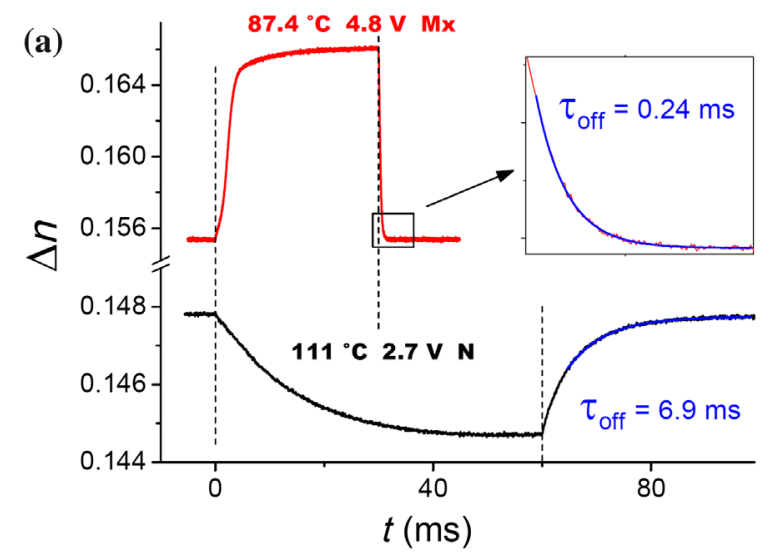

observed birefringence, $\Delta n=n_{m m}-n_{k k}$, is small. On the contrary, when planar $M_{X}$ domains are grown without the field, $\mathbf{n}$ is oriented parallel to $\mathbf{r}$, and this orientation defines that of the smectic layers. Under realistically strong fields, $\mathbf{n}$ keeps its initial orientation, but, due to the out-ofplane reorientation of $\mathbf{m}$, the birefringence increases with the field. At high applied voltage $U, \mathbf{m}$ aligns parallel to the field and $\Delta n(U)$ saturates to $n_{n n}-n_{k k}$ [Fig. 6(b)]. The extrapolated birefringence value is close to that expected by the Haller fit [Fig. 6(a)], and its temperature variation is very weak.

The characteristic times of the optical response of the BFrTr in the $M_{X}$ phase are compared with those of the usual

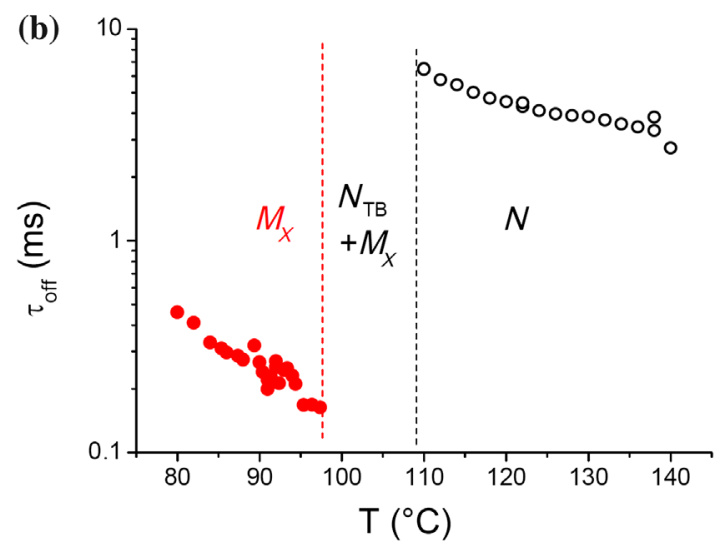

FIG. 7. Time evolution of the optical response during the Fréedericksz transition in the $N$ (FrTr, black lines and symbols) and $M_{X}$ (BFrTr, red lines and symbols) phases of the BP12 mixture (cell thickness $d=1.4 \mu \mathrm{m}$ ). (a) Optical response to dc pulses ( $U>U_{c}$; the dashed straight lines mark the start and the end of the pulse). The relaxation time $\tau_{\text {off }}$, which depends only on the cell thickness, is much faster in the $M_{X}$ phase. Fits of the data with an exponential relaxation law are shown in blue. (b) Temperature dependence of $\tau_{\text {off }}$ in the $N$ (open black symbols) and $M_{X}$ (full red symbols) phases. 

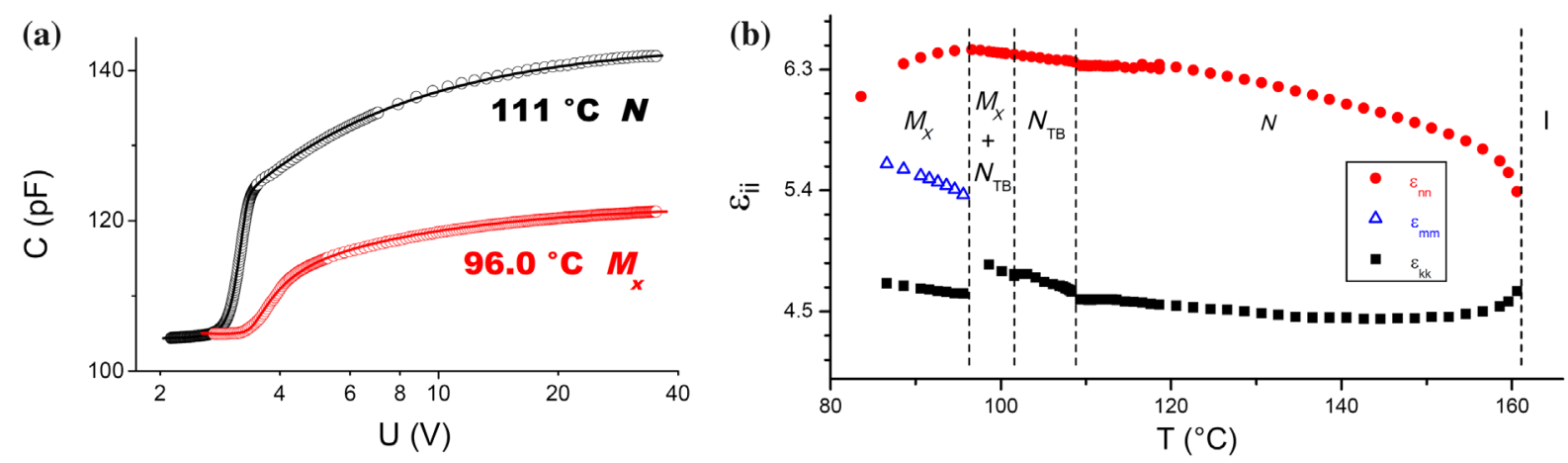

FIG. 8. Dielectric data of the BP12 mixture (cell thickness $9.8 \mu \mathrm{m}$ ). (a) Cell capacitance versus applied voltage in the $N$ phase (black symbols) and in the $M_{X}$ phase (red symbols). The solid lines show the best fits of the data with the theoretical model (see Appendix E). (b) Temperature dependence of the dielectric tensor eigenvalues measured in the uniaxial $N$ and $N_{\text {TB }}$ phases and in the biaxial $M_{X}$ phase.

FrTr in the $N$ phase in Fig. 7(a). The on time $\tau_{\text {on }}$ is proportional to $E^{-2}$ and, therefore, becomes very small for large fields. On the contrary, the off time $\tau_{\text {off }}$ of the response when the field is switched off is independent of the field. The temperature dependence of $\tau_{\text {off }}$ in the $N$ phase [Fig. 7(b)] is typical, as it just increases from approximately $3 \mathrm{~ms}$ at high temperature to approximately $8 \mathrm{~ms}$ close to the $N_{\text {TB }}$ phase. However, quite strikingly, $\tau_{\text {off }}$ of the BFrTr in the $M_{X}$ phase is much faster, by almost 2 orders of magnitude when extrapolated to the same temperature.

\section{Dielectric experiment}

The dielectric technique is complementary to the birefringence measurements. The cell capacitance $C(U)$, measured with various surface- and field-induced alignments (see Appendix C), provided all three eigenvalues, $\varepsilon_{k k}, \varepsilon_{n n}$, and $\varepsilon_{m m}$, of the dielectric tensor $\varepsilon$ (see Appendix E). The capacitance, measured in the $N$ and $M_{X}$ phases, varies smoothly and reversibly with the voltage [Fig. 8(a)], through the field-induced FrTr and BFrTr, respectively. Fits of these data with theoretical models provide two of the $\varepsilon$ components: $\varepsilon_{\perp}$ and $\varepsilon_{\|}$in the $N$ phase [58-60] and $\varepsilon_{k k}$ and $\varepsilon_{m m}$ in the $M_{X}$ phase. The third component, $\varepsilon_{n n}$, of the $M_{X}$ phase is measured in a field-induced quasihomeotropic domain. In the $N_{\mathrm{TB}}$ phase, the components $\varepsilon_{\perp}=\varepsilon_{k k}=\varepsilon_{m m}$ and $\varepsilon_{\|}=\varepsilon_{n n}$ are measured at fixed voltages because of the absence of FrTr in this phase. The temperature dependence of the dielectric tensor components across the three mesophases is presented in Fig. 8(b), which provides further direct evidence for the biaxiality of the $M_{X}$ phase: $\varepsilon$ has only two different components, $\varepsilon_{\perp}$ and $\varepsilon_{\|}$, in the uniaxial $N$ and $N_{\text {TB }}$ phases, whereas $\varepsilon_{\perp}$ is split in two different components, $\varepsilon_{k k} \neq \varepsilon_{m m}$, in the $M_{X}$ phase. The dielectric data are further exploited in the next section.

\section{DISCUSSION}

\section{A. Nature of the $M_{X}$ phase}

The whole set of $x$-ray scattering, dielectric, and electrooptic data shows that the $M_{X}$ phase is an orthogonal smectic with liquidlike layers, i.e., a smectic $A$, with primary director $\mathbf{n}$ parallel to the smectic wave vector $\mathbf{q}_{0}$ (see Appendix F for more details). During the $N_{\mathrm{TB}}-M_{X}$ phase transition, $\mathbf{q}_{0}$ appears parallel to the helix axis $\mathbf{h}$ of the $N_{\text {TB }}$ modulation. In the $M_{X}$ single domains, $\mathbf{n}$ remains parallel to $\mathbf{h}$ and, therefore, parallel to $\mathbf{q}_{0}$. Finally, since the order tensor of the $N$ and $M_{X}$ phases is uniform in the absence of a field, the slowest axis $\mathbf{N}$ in these phases has the same orientation and is parallel to $\mathbf{n}$. Qualitatively, the BFrTr also confirms that the $M_{X}$ phase is $\operatorname{Sm} A$ and not $\mathrm{SmC}$. Indeed, the variation of $\Delta n(U)$ occurs without reorientation of the slow axis $\mathbf{N}$, because the order tensor $\mathbf{Q}$ rotates around its main axis $\mathbf{n}$, which remains parallel to $\mathbf{q}_{0}$. Moreover, the variation of both $\Delta n(U)$ and $\Delta \varepsilon(U)$ during the transition clearly indicates that the order tensor $\mathbf{Q}$ is biaxial.

In principle, various microscopic structures differing by the in-layer order of the tilted monomers are possible for the intercalated $\operatorname{Sm} A$ phase of bent-shaped dimers. If the tilt direction were continuously degenerated, the monomers would form a de Vries-SmA-like structure, but it is uniaxial and, therefore, cannot explain the observed biaxiality of the $M_{X}$ phase. Another possible structure has doubly degenerated tilt of the monomers within each smectic layer (Fig. 25). However, although this structure is biaxial, it should be ruled out, because it does not minimize the interaction energy of the monomers (see Appendix F for more details). Consequently, we identify the structure of the $M_{X}$ phase as that shown in Fig. 3, which is an intercalated biaxial $\operatorname{Sm} A$ phase of the bent-shaped dimers with anticlinic $\mathrm{Sm} C_{A}$ organization of the monomer units. One may find this structure quite reminiscent of that of the antiferroelectric $\operatorname{Sm} A_{\mathrm{PA}}$ phase $[14,15,42]$. However, the $\operatorname{Sm} A_{\mathrm{PA}}$ phase is not intercalated, and its fluid smectic layers are just formed by the bent-shaped molecules oriented with their main axis normal to the layer and their transverse dipoles parallel to one another. Therefore, the phase is a biaxial smectic $A$ with polar in-layer order. The direction of the polarization alternates from one layer to the next, and, hence, the phase is antiferroelectric. Consequently, in each 
layer, the polarization vector aligns parallel to an applied moderate in-plane electric field, resulting in the macroscopic polarization of the phase.

In contrast, the $M_{X}$ phase displays no polar response under the applied field. Moreover, the polar electroclinic effect [23] observed in the $N_{\mathrm{TB}}$ completely disappears in the $M_{X}$ phase, which behaves as a purely dielectric material. This result is due to the complete intercalation of the dimers that locks the polarization of any monomer layer to those of its first neighbors, thus effectively suppressing any ferroelectric response, as already recognized in Ref. [10]. So, hereafter, following the notations of Ref. [42], we refer to the $M_{X}$ phase as $\operatorname{Sm} A_{b}$. (Note that the $\operatorname{Sm} A_{b}$ phase is expected and has been reported [12] for strongly biaxial boardlike molecules; it is sometimes also labeled $\mathrm{SmC}_{M}$ [51], in honor of William McMillan.)

\section{B. Elasticity of the $\operatorname{Sm} A_{b}$ phase}

Our explanation of the striking electro-optic behavior of $\operatorname{Sm} A_{b}$ relies on its biaxial physical properties and elastic response. As for any smectic phase, there are two contributions to the elastic energy of $\operatorname{Sm} A_{b}$. The first one is related to the orientational order of the phase. For a uniaxial phase, this "nematiclike" elasticity is given by the usual Frank expansion of the distortion energy in the director derivatives (here and in the following, we neglect the surfacelike terms, which are not relevant in our case) [61]:

$$
f^{n}=\frac{1}{2}\left[K_{11}^{n}\left(\boldsymbol{s}^{n}\right)^{2}+K_{22}^{n}\left(t^{n}\right)^{2}+K_{33}^{n}\left(\boldsymbol{b}^{n}\right)^{2}\right] .
$$

Here, the vectors $\boldsymbol{s}^{n}=\boldsymbol{n}(\nabla \cdot \boldsymbol{n})$ and $\boldsymbol{b}^{n}=\boldsymbol{n} \times(\nabla \times \boldsymbol{n})$ and the pseudoscalar $t^{n}=\boldsymbol{n} \cdot(\nabla \times \boldsymbol{n})$ describe the main distortion modes, respectively, splay, bend, and twist, of the (primary) nematic director $\mathbf{n}$ and the $K_{i i}^{n}$ are the Frank elastic constants related to the derivatives of $\mathbf{n}$. When the orientational order is biaxial, as in the $\operatorname{Sm} A_{b}$ phase (and in biaxial nematics), the distortion energy is much more complex, because it has additional terms, which are related to the derivatives of the secondary director $\mathbf{m}$ :

$$
f^{m}=\frac{1}{2}\left[K_{11}^{m}\left(\boldsymbol{s}^{m}\right)^{2}+K_{22}^{m}\left(t^{m}\right)^{2}+K_{33}^{m}\left(\boldsymbol{b}^{m}\right)^{2}\right],
$$

where $\boldsymbol{s}^{m}=\boldsymbol{m}(\nabla \cdot \boldsymbol{m}), \boldsymbol{b}^{m}=\boldsymbol{m} \times(\nabla \times \boldsymbol{m})$, and $t^{m}=\boldsymbol{m}$. $(\nabla \times \boldsymbol{m})$ describe the splay, bend, and twist of $\mathbf{m}$, respectively, and the $K_{i i}^{m}$ are the corresponding Frank elastic constants. Additional cross terms between $\mathbf{m}$ and $\mathbf{n}$ and their gradients result in a very complex biaxial-nematic elastic energy containing 12 independent terms (for achiral molecules) $[62,63]$.

The second contribution to the elastic energy of the $\operatorname{Sm} A_{b}$ phase (as of any other smectic) is a "smecticlike" term related to the distortion of the positional ordering, i.e., to the compression and deformation of the layers [51]. Actually, this energy is prohibitively large compared to the moderate electric energy involved in our experiments, and such distortion of the layers should be negligible. Indeed, our observations confirm that no reorganization of the layers occurs in the $\operatorname{Sm} A_{b}$ phase, even when strong fields are applied.

The rigid smectic elasticity of $\operatorname{Sm} A_{b}$ substantially simplifies the elastic response to the electric field. Indeed, the bend and twist of $\mathbf{n}$ are forbidden, because $\operatorname{Sm} A_{b}$ is an orthogonal smectic [51]. Then, the only possible distortion of $\mathbf{n}$ is the splay. However, it is coupled with the bend of the layers, and its variation requires reorganizing the layers at large scale, which is energetically costly. No such reorganization is observed in the $\operatorname{Sm} A_{b}$ domains that we study, which confirms that $\mathbf{n}$ remains uniform during the BFrTr. Consequently, the elastic energy of $\operatorname{Sm} A_{b}$ just reduces to Eq. (2), which provides an analogy between the elastic distortions of the $\mathbf{n}$ director in the $N$ phase and those of the $\mathbf{m}$ director in the $\operatorname{Sm} A_{b}$ phase (Fig. 9).

Moreover, the boundary conditions $\mathbf{m} \perp \mathbf{r}$ are uniform in the plane of the cell, and the field is applied along the cell normal, $\mathbf{E} \| \mathbf{z}$. Therefore, the distortion of $\mathbf{m}$ is onedimensional $[\mathbf{m}=\mathbf{m}(z)]$ and planar $[\mathbf{m}(z) \perp \mathbf{r}]$, with only splay and bend but no twist (Fig. 10). This distortion geometry is again an exact analog of the usual FrTr in the splay geometry [51]. Under the field, the free energy in the $N$ and $\operatorname{Sm} A_{b}$ phases becomes, respectively,

$$
\begin{gathered}
f^{n}=\frac{1}{2}\left\{\left[K_{11}^{n} \sin ^{2} \theta+K_{33}^{n} \cos ^{2} \theta\right]\left(\frac{d \theta}{d z}\right)^{2}+\boldsymbol{D} \cdot \boldsymbol{E}\right\}, \\
f^{m}=\frac{1}{2}\left\{\left[K_{11}^{m} \sin ^{2} \theta+K_{33}^{m} \cos ^{2} \theta\right]\left(\frac{d \theta}{d z}\right)^{2}+\boldsymbol{D} \cdot \boldsymbol{E}\right\},
\end{gathered}
$$

where $\theta=\theta(z)$ is the angle between the relevant director (respectively, $\mathbf{n}$ or $\mathbf{m}$ ) and the $\mathbf{z}$ axis, $\mathbf{E}=[0,0, E(z)]$ is the electric field in the cell, which varies with $z$ due to the director distortion, and the relevant $D_{z}$ component of the dielectric displacement $\mathbf{D}$ is $D_{z}=\varepsilon_{z z} E_{z}=$ const, where $\varepsilon_{z z}=\varepsilon_{\perp} \sin ^{2} \theta+\varepsilon_{\|} \cos ^{2} \theta$ in the uniaxial $N$ case and $\varepsilon_{z z}=\varepsilon_{k k} \sin ^{2} \theta+\varepsilon_{m m} \cos ^{2} \theta$ in the $\operatorname{Sm} A_{b}$ case.

Thanks to this analogy, we can easily describe the behavior of the $\operatorname{Sm} A_{b}$ phase under a field by using the well-known solutions [58-60,64-66] for the FrTr in the $N$ phase. The voltage threshold of the transition, $U_{c}^{i}$, is given by

$$
U_{c}^{n}=\pi \sqrt{\frac{K_{11}^{n}}{\varepsilon_{0}\left(\varepsilon_{\|}-\varepsilon_{\perp}\right)}} ; \quad U_{c}^{m}=\pi \sqrt{\frac{K_{11}^{m}}{\varepsilon_{0}\left(\varepsilon_{m m}-\varepsilon_{k k}\right)}}
$$

(where $i=n, m$ indicates the director involved in the transition).

Here, $\varepsilon_{0}$ is the vacuum permittivity, and $\left(\varepsilon_{\|}-\varepsilon_{\perp}\right)$ and $\left(\varepsilon_{m m}-\varepsilon_{k k}\right)$ are the effective dielectric anisotropies in the $N$ and $\operatorname{Sm} A_{b}$ phases, respectively. Up to $U=U_{c}^{i}$, the field-induced torque on the relevant director is weaker 


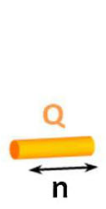

$\mathbf{s}^{n}=\mathbf{n}(\boldsymbol{\nabla} \cdot \mathbf{n}) \neq 0$

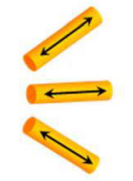

Splay

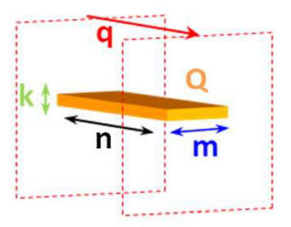

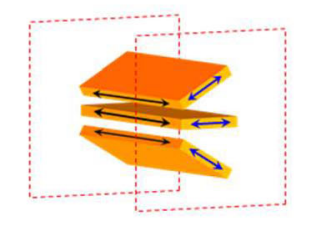

$\mathbf{s}^{m}=\mathbf{m}(\boldsymbol{\nabla} \cdot \mathbf{m}) \neq 0$ $t^{n}=\mathbf{n} \cdot(\boldsymbol{\nabla} \times \mathbf{n}) \neq 0$

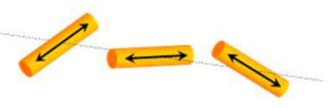

Twist

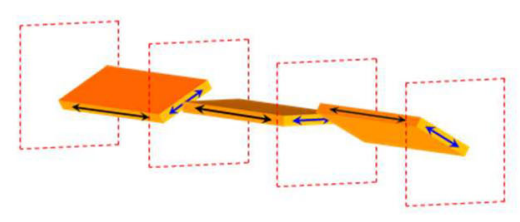

$t^{m}=\mathbf{m} \cdot(\boldsymbol{\nabla} \times \mathbf{m}) \neq 0$ $\mathbf{b}^{n}=\mathbf{n} \times(\boldsymbol{\nabla} \times \mathbf{n}) \neq 0$

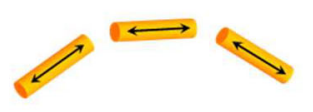

Bend

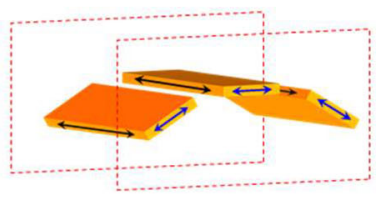

$\mathbf{b}^{m}=\mathbf{m} \times(\boldsymbol{\nabla} \times \mathbf{m}) \neq 0$

FIG. 9. Schematic representation of the nematic directors $(\mathbf{n}, \mathbf{m}$, and $\mathbf{k})$, the order parameter tensor (Q), and the main distortion modes in the $N$ and $\operatorname{Sm} A_{b}$ phases. In the uniaxial $N$ phase (top row), the only relevant distortions are those of the main director $\mathbf{n}$. In the $\operatorname{Sm} A_{b}$ phase (bottom row) and at fixed structure of the smectic layers (shown in red), the only relevant distortions are those of the secondary director $\mathbf{m}$.

than the surface-anchoring torque, and the cell texture remains undistorted. Above the threshold, the director field is distorted and the field dependence of its tilt angle, $\theta(z)$, is described by the same expressions in the $\operatorname{Sm} A_{b}$ as in the $N$ phase but involving the material constants related to $\mathbf{m}$ instead of those related to $\mathbf{n}$.

The analysis of the dielectric data provides a wealth of information about the elastic and surface-anchoring properties of the $N$ and $\operatorname{Sm} A_{b}$ phases of the BP12 mixture (see Appendix E for details). The excellent fit of the data with the theoretical model [Fig. 8(a)] gives $U_{c}^{i}$ for both phases (Fig. 11). In the $N$ phase, upon cooling, we observe the usual weak variation of $U_{c}^{n}(T)$, which increases from approximately 2 to approximately $3 \mathrm{~V}$ through the $50^{\circ} \mathrm{C}$
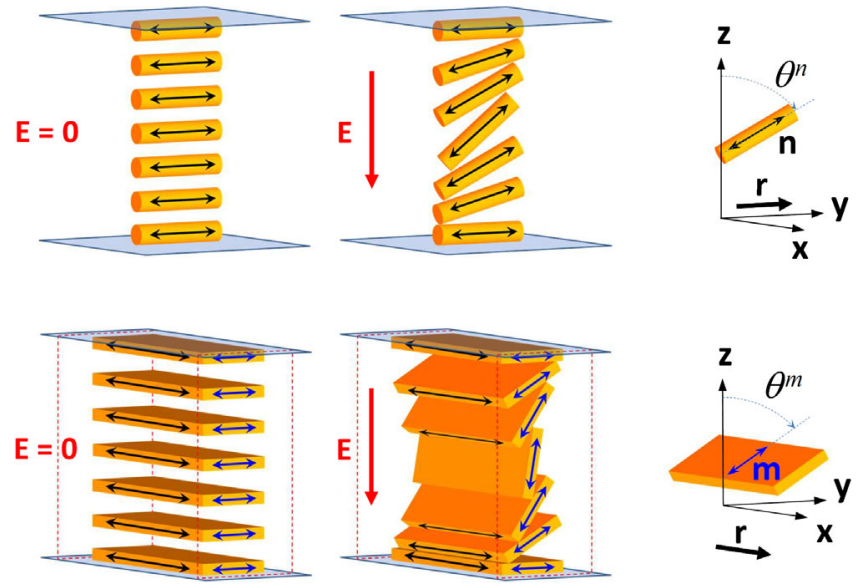

FIG. 10. Geometry of the distortions of $\mathbf{n}$ in the $N$ phase (top row) and $\mathbf{m}$ in the $\operatorname{Sm} A_{b}$ phase (bottom row); in the left and middle columns, the applied voltage is, respectively, below and above the Fréedericksz transition threshold. $\mathbf{r}$ is the rubbing direction, $(\mathbf{x}, \mathbf{y}, \mathbf{z})$ is a reference frame, $\theta^{n}$ (respectively, $\left.\theta^{m}\right)$ is the angle of the $\mathbf{n}$ (respectively, $\mathbf{m}$ ) director with the normal to the cell ( $\mathbf{z}$ axis). The smectic layers are shown in red. nematic range of $\mathrm{BP} 12$. In $\operatorname{Sm} A_{b}$, the threshold is only slightly higher $\left[3.5 \mathrm{~V}<U_{c}^{m}(T)<4 \mathrm{~V}\right]$, which is quite counterintuitive, because the structure of this phase is expected to be much more rigid than the nematic one. However, this low threshold value can be explained by the fact that the BFrTr induces only a distortion of $\mathbf{m}$, which does not involve the rigid smectic order.

The temperature dependence (Fig. 12) of the splay elastic constants $K_{11}^{n}$ and $K_{11}^{m}$ (in the $N$ and $\operatorname{Sm} A_{b}$ phases, respectively) is obtained directly from Eq. (5) and from the data in Figs. 8 and 11. $K_{11}^{n}$ increases significantly with decreasing temperature, which is the usual behavior for the nematic phase. As for many other $N_{\mathrm{TB}}$-forming compounds $[55,56,67,68]$, this behavior is not influenced by the

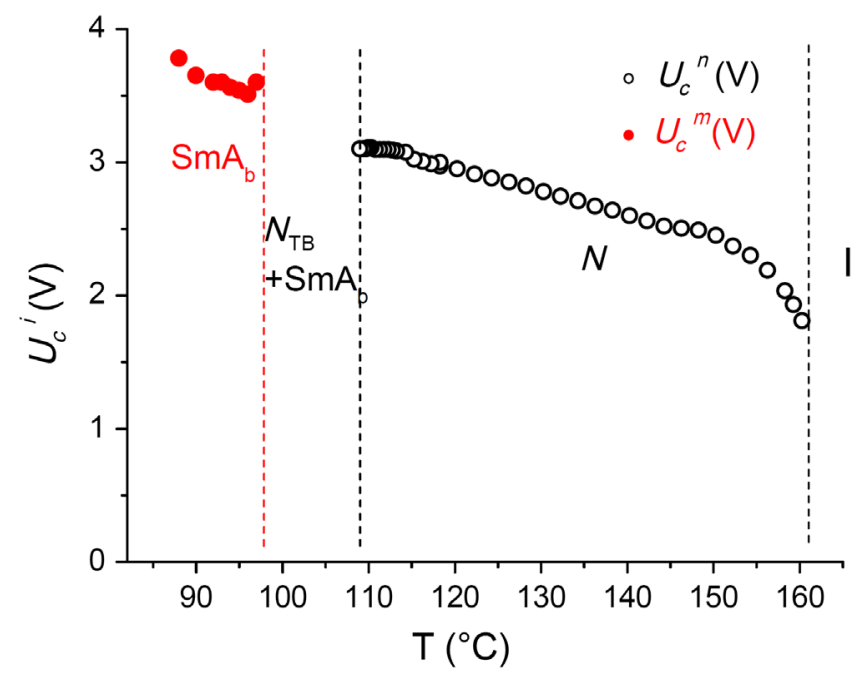

FIG. 11. Temperature dependence of the Fréedericksz transition threshold in the $N$ and $\operatorname{Sm} A_{b}$ phases. (There is no Fréedericksz transition in the $N_{\mathrm{TB}}$ phase, and no data are available for the biphasic $N_{\mathrm{TB}} / \mathrm{Sm} A_{b}$ range due to the nonuniform cell texture.) 

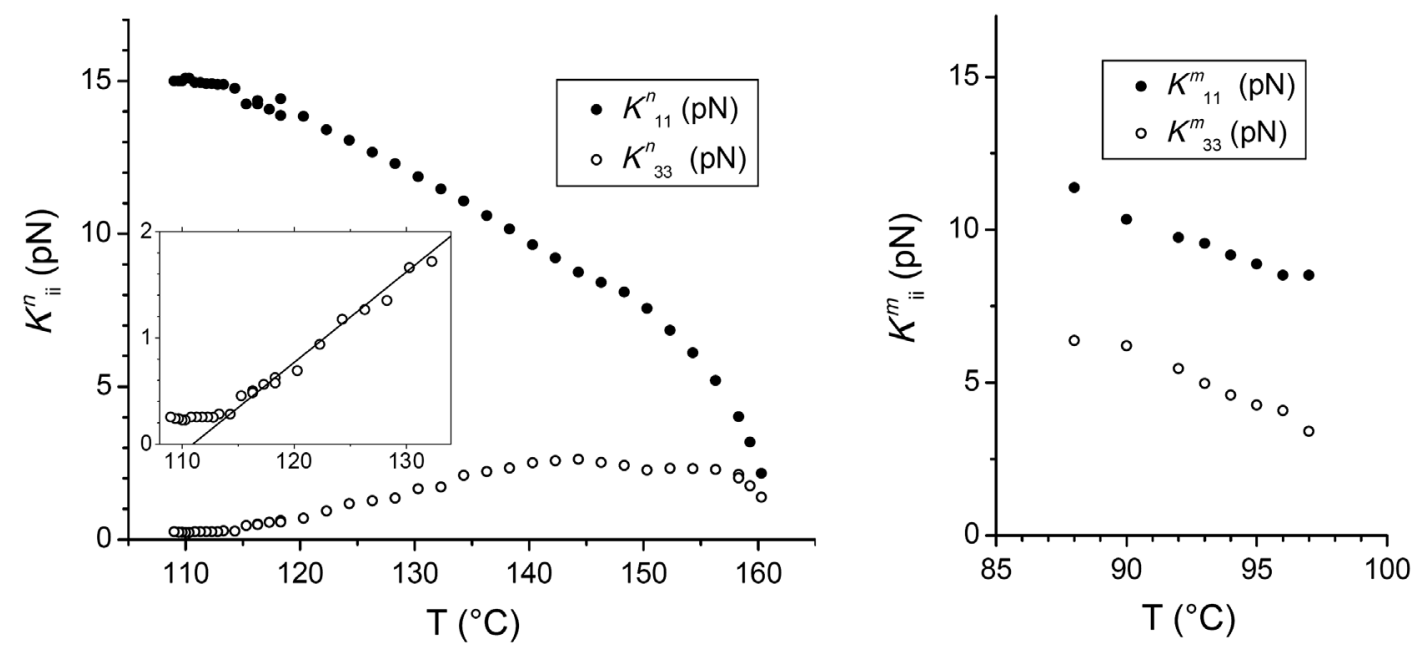

FIG. 12. Temperature dependence of the splay and bend elastic constants in the $N$ (a) and $\operatorname{Sm} A_{b}$ (b) phases of the BP12 mixture. [The inset in (a) shows a magnification of the $K_{33}^{n}$ curve.]

presence of the $N_{\text {TB }}$ phase. The order of magnitude of $K_{11}^{n}$, in the piconewton range, is also typical for both rodlike and bent-shaped nematics. In contrast, the bend elastic constant $K_{33}^{n}$ decreases strongly and almost linearly with decreasing temperature, reaching values much smaller than $K_{11}^{n}$, which is the theoretically predicted [17,24] and experimentally observed $[55,56,67,68]$ pretransitional behavior in the $N$ phase preceding the $N_{\mathrm{TB}}$ phase. This feature is related to the pathological bend elasticity of the bent-shaped nematogen molecules, which leads to the spontaneous bend of $\mathbf{n}$ in the $N_{\text {ТВ }}$ phase [17].

The behavior of $K_{11}^{m}$ and $K_{33}^{m}$ in the $\operatorname{Sm} A_{b}$ phase is less peculiar than that of $K_{33}^{n}$. Their values are slightly smaller than the usual $K_{i i}^{n}$ values in the $N$ phase, and they increase with decreasing temperature. $K_{33}^{m}$ is smaller than $K_{11}^{m}$, but they remain of the same order of magnitude. In brief, $K_{33}^{m}$ in the $\operatorname{Sm} A_{b}$ phase has a much more regular behavior than that of $K_{33}^{n}$ in the $N$ phase. The reason why is that the elasticity of the $\mathbf{m}$ director is not related to the bent shape of the molecules but essentially to their biaxiality (indeed, a $\operatorname{Sm} A_{b}$ phase formed of lathlike molecules should have a similar elastic behavior). Moreover, the elasticity of the $\mathbf{m}$ director in the $\operatorname{Sm} A_{b}$ phase is drastically different from that of the $\mathbf{n}$ director in usual $\operatorname{Sm} A$ phases. Indeed, since $\mathbf{n}$ is parallel to the layer normal, its bend is forbidden because of its incompatibility with the layered structure, resulting in the divergence of $K_{33}^{n}$. In contrast, $K_{11}^{n}$ remains finite but is larger than the splay modulus of the $N$ phase, because the splay is coupled to the curvature of the layers, which costs additional elastic energy. However, in the $\operatorname{Sm} A_{b}$ phase, $\mathbf{m}$ is parallel to the layers instead of their normal, and the curvature of $\mathbf{m}$ is then possible without any layer distortion; i.e., the (re)orientation of $\mathbf{m}$ is decoupled from the smectic order. This result explains why the elasticity of the $\mathbf{m}$ director is nematiclike in $\operatorname{Sm} A_{b}$ and why, contrary to the usual $\operatorname{Sm} A$, the BFrTr is then possible.

\section{Reorientation dynamics}

One of the most striking features of the BFrTr is its very short relaxation time. This fast relaxation rate in the $\operatorname{Sm} A_{b}$ phase is due to the collective rotation of the molecules around their long axes rather than around their short axes, as is usually the case in the $N$ phase. The theoretical description of the rotational viscosities $\gamma_{1}^{n}$ and $\gamma_{1}^{m}$ for reorientation, respectively, of the primary $\mathbf{n}$ and secondary $\mathbf{m}$ directors in a biaxial phase is a complex task [63,69-71]. However, one can qualitatively expect that $\gamma_{1}^{m} \ll \gamma_{1}^{n}$, owing to the significantly different molecular cross sections involved in the two rotations. Figure 13 shows the temperature dependence of $\gamma_{1}^{n}$ and $\gamma_{1}^{m}$ deduced from the measured relaxation times and elastic moduli by a procedure well known for the FrTr case [72] and extended here for the BFrTr case (see Appendix D). Both viscosities increase

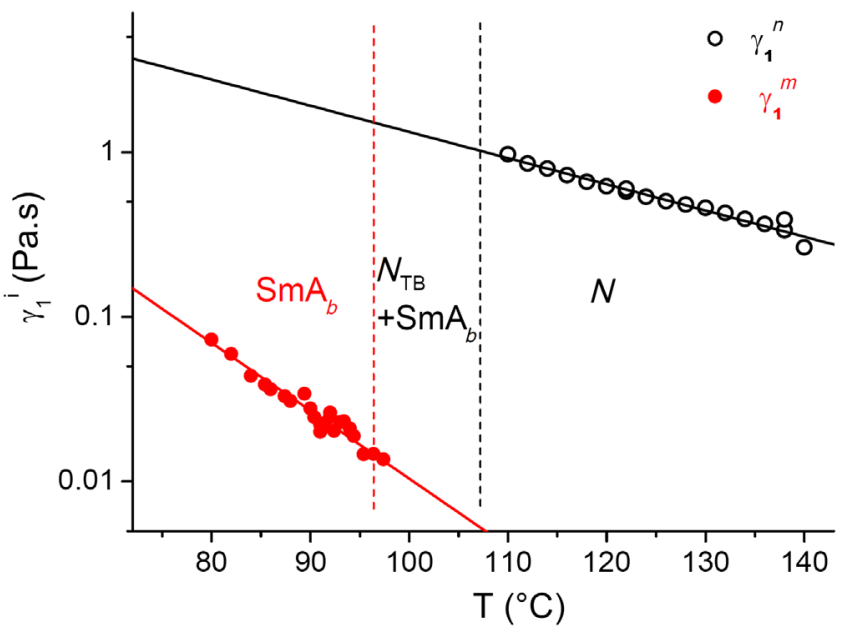

FIG. 13. Rotational viscosities of the BP12 mixture for reorientation of $\mathbf{n}$ in the $N$ phase (open black symbols) and $\mathbf{m}$ in the $\operatorname{Sm} A_{b}$ phase (full red symbols). The solid straight lines show exponential decay fits of the viscosities (Arrhenius-law behavior). 
exponentially with decreasing temperature, but $\gamma_{1}^{m}$ is much smaller than $\gamma_{1}^{n}$ (by about 2 orders of magnitude when extrapolated to the same temperature). We note that, although some anisotropy of the rotational viscosities is theoretically expected for biaxial nematics [70,71], the ratio $\gamma_{1}^{n} / \gamma_{1}^{m} \sim 100$ observed here is much larger than the predicted one $(<10)$. Furthermore, counterintuitively, the activation energy for $\gamma_{1}^{m}$ is twice as large as that for $\gamma_{1}^{n}$ despite the much smaller steric hindrance for rotation of the molecule around its long axis (which is involved in the $\gamma_{1}^{m}$ case). However, the rotational viscosity describes the collective rotation of all the molecules and, for this reason, depends strongly on the relevant order parameter components $\mathbf{Q}_{m m}$ and $\mathbf{Q}_{n n}$. Further detailed studies of the temperature dependence of the biaxial order tensor are, therefore, needed to clarify the observed temperature dependence of the rotational viscosities.

\section{Comparison with other smectics}

The field-induced transition in the $\operatorname{Sm} A_{b}$ phase is an exact analog of the Fréedericksz transition in nematics. Its existence is closely related to the biaxiality of this smectic phase. Indeed, in the uniaxial $\operatorname{Sm} A$ phase, the reorientation of the $\mathbf{n}$ director is impossible without dilation of the smectic layers, which is energetically very costly. For this reason, the field-induced transition in $\operatorname{Sm} A$ is only a "ghost" transition [3]; i.e., it is practically unobservable because of its negligible amplitude. A similar behavior is also expected for the $N_{\mathrm{TB}}$ phase, whose macroscopic symmetry is the same as that of the chiral $\operatorname{Sm} A$ [73], as it is pseudolayered and uniaxial. The case of the $\mathrm{SmC}$ phase is more complex. Because of its biaxiality, two types of field-induced transitions are possible in different geometries [3]: ghost transitions with negligible amplitude due to the dilation of the layers, as in the SmA, and nematiclike transitions with finite amplitude but with rotation of the $\mathbf{n}$ director confined on a cone. Although the physical mechanism in this second case is similar to the FrTr in nematics, its optical behavior is very different, and this effect has not yet found any practical application. Optically, $\mathrm{SmC}$ is approximately uniaxial with optic axis $\mathbf{n}$. Therefore, during the transition, the optic axis rotates on a cone instead of in a plane for the nematic and $\operatorname{Sm} A_{b}$ phases. Hence, the main optical effect during the transition is not the variation of the birefringence but instead a twist of the texture and in-layer rotation of the optic axis by a limited angle, smaller than the $\mathrm{SmC}$ tilt angle. Moreover, the relaxation time of the FrTr in the $\mathrm{SmC}$ phase should be slow, as in the nematic phase, because the transition involves the reorientation of the long molecular axis. In contrast, the very fast reorientation of the short molecular axis, observed here in the BFrTr of the $\operatorname{Sm} A_{b}$ phase, is much more promising for practical applications.

Fast field-induced electro-optic effects have been reported earlier in the uniaxial $N$ and $\operatorname{Sm} A$ phases of bent-shaped liquid crystals. They are due either to a field- induced biaxiality of the microscopic order parameter of the phase [74] or to a field-induced alignment of cybotactic (smectic) clusters in the $N$ phase [75] (i.e., a field-induced biaxiality of the macroscopic order parameter). In contrast to the FrTr reported here, in both these cases, the field induces a biaxial order instead of reorienting a preexistent biaxial order parameter. Faster and thresholdless on and off switchings are expected in these cases under strong fields (approximately $10 \mathrm{~V} / \mu \mathrm{m}$ ), but, surprisingly, the reported relaxation times (approximately $1 \mathrm{~ms}$ ) are 4 times slower than the relaxation time $\tau_{\text {off }} \sim 240 \mu$ s of the BFrTr in the $\operatorname{Sm} A_{b}$ phase [Fig. 7(a)].

The BFrTr observed here with the intercalated $\operatorname{Sm} A_{b}$ is also expected with other orthogonal biaxial smectics, e.g., with nonintercalated $\operatorname{Sm} A_{b}$ phases [12]. Although here we observe the BFrTr in a $\operatorname{Sm} A_{b}$ phase formed by bent-shaped dimer molecules and preceded by the $N_{\mathrm{TB}}$ phase, the same electro-optic effect should be expected for $\operatorname{Sm} A_{b}$ phases formed by a much larger class of mesogenic compounds, e.g., for bent-core or even boardlike molecules. Moreover, some modulated nematic phases, like the elusive $N_{\text {SB }}$ phase $[17,24,31]$ and the recently discovered splay nematic [27,76-78], are biaxial and pseudolayered and should present a BFrTr identical to that reported here.

\section{CONCLUSION}

By adding a small amount of a suitable rodlike nematogenic molecule to the BNA-76 bent-shaped dimer, we stabilize the $M_{X}$ phase against crystallization, which allows for its structural and electro-optic investigation. The x-ray scattering study shows that the $M_{X}$ phase is a smectic phase with liquidlike layers, resulting from the complete intercalation of the dimer molecules, a structure already reported for other bent-shaped dimers [7,9-11,32-36,38-42]. Moreover, multiple observations of the textures and the electro-optic response of single domains reveal that the $M_{X}$ phase is biaxial and lacks ferroelectric order. We therefore identify it as a $\operatorname{Sm} A_{b}$ phase. The monomer mesogenic moieties of the dimer molecules form smectic layers with each dimer molecule spanning over two neighboring layers. In each layer, the monomer moieties are tilted in the same way, as in a smectic $C$, but the tilt direction alternates in adjacent layers, as in the $\mathrm{SmC}_{A}$ phase. The primary director $\mathbf{n}$ of the phase is normal to the smectic layers, whereas the secondary director $\mathbf{m}$ is parallel to the layers.

Surprisingly, the $\operatorname{Sm} A_{b}$ phase displays a counterintuitive, nematiclike, electro-optic effect, with moderate threshold voltage and fast relaxation time. We show that this effect is an analog of the Fréedericksz transition in the $N$ phase, but with reorientation of $\mathbf{m}$, at fixed orientation of $\mathbf{n}$. Because of the smectic constraints imposed to $\mathbf{n}$, the elastic behavior of $\operatorname{Sm} A_{b}$ is here much simpler than the complex one of biaxial nematics. By analogy with the $N$ phase, we show that the nematic elastic energy of $\operatorname{Sm} A_{b}$ is Frank-like but with elastic moduli $K_{i i}^{m}$ related to the splay, twist, and bend 
distortions of $\mathbf{m}$. Using this analogy for the interpretation of our experimental results, we show that the bend and splay moduli for $\mathbf{m}$ in the $\operatorname{Sm} A_{b}$ phase are of the same order of magnitude as their analogs for $\mathbf{n}$ in the nematic phase (far from the $N-N_{\text {TB }}$ transition). Similarly, we deduce from the dynamic behavior of the BFrTr that the rotational viscosity constant for reorientation of $\mathbf{m}$ is almost 2 orders of magnitude smaller than that in the uniaxial nematic.

We present here an original experimental investigation of the elastic and electro-optic response of the biaxial order parameter tensor in a thermotropic liquid crystal. It opens the way for similar studies of other biaxial smectics, of the biaxial nematic, and also of the splay-bend nematic. Indeed, this latter, pseudolayered, phase also has biaxial symmetry, and, even though its pitch should be larger than the dimer length, it should nevertheless show exactly the same electro-optic behavior as that of $\operatorname{Sm} A_{b}$.

In this work, we study a dimer molecule with a markedly bent shape, leading to an $N_{\text {TB }}$ phase in addition to the $\operatorname{Sm} A_{b}$ one. However, the remarkable biaxial elasticity of $\operatorname{Sm} A_{b}$ is not due to the bent shape of the molecules. Therefore, similar elastic and electro-optic behavior should also be expected with phases formed by weakly bent-shaped dimers, bentcore molecules, and even strongly biaxial board-shaped molecules. We hope that our work will, therefore, stimulate further interest in the synthesis of new molecules presenting biaxial mesophases and in the investigation of their elastic properties and electro-optic effects.

The biaxial Fréedericksz transition is very promising for practical applications. Indeed, the synthesis of the BNA-76 compound is not particularly involved or expensive, and it may readily be up-scaled. However, several important improvements are still required for applications: better surface-anchoring alignment methods for easy and reproducible production of large uniform domains; wider temperature range of the phase, centered around room temperature; new optical geometries taking advantage of the increase of the birefringence under a field, as opposed to its decrease in the nematic phase, and of the optical biaxiality of the $\operatorname{Sm} A_{b}$ phase; and optimization of the switching characteristics, such as the threshold voltage and the relaxation time. This exciting roadmap is certainly worth exploring, because the threshold voltage of the biaxial Fréedericksz transition is similar to the usual values in the nematic phase and its response times are much faster. Therefore, the biaxial electric-field response of $\operatorname{Sm} A_{b}$ could be a good candidate for replacing the traditional uniaxial-nematic electro-optic effects in fast-switching devices. We note that even at the present stage, before any expected improvement of the materials is achieved, the $\mathrm{Sm} A_{b}$ phase of the BP12 mixture can already be useful in thermostated, nondisplay, electrooptic devices for which fast response is a key feature, such as modulators, switches, wave plates with continuous electric control of the retardation, light-beam deflectors, devices for adaptive polarization control, etc.

\section{ACKNOWLEDGMENTS}

A. K., I. Dokli, and A. L. thank the Croatian Science Foundation (Grant No. IP-2019-04-7978) and Ruđer Bošković Institute for financial support. This work was supported by the Agence Nationale pour la Recherche ANR (France) through Grant BESTNEMATICS, No. ANR-15-CE24-0012, by the French-Croatian bilateral program COGITO, and by the Université de Picardie Jules Verne, Amiens, France. This research was supported by the $\mathrm{Hu}$ Foundation, California State University, Sacramento. Special thanks to Department of Physics and Astronomy, California State University, Sacramento for partially covering of publication cost. The authors thank G. R. Luckhurst, T. Sergan, M. Nobili, and C. Blanc for helpful discussions and D. Petermann for technical assistance with the X-ray scattering experiments.

Note added in proof.-Recently, another paper reporting a new, quite convincing, example of the $\operatorname{Sm} A_{b}$ phase was published [79]. This paper also describes some electrooptic experiments that show an electric-field-induced increase of the birefringence in that phase. However, in Ref. [79] this effect was not investigated and analyzed in detail as it was not the main focus of this paper.

\section{APPENDIX A: MATERIALS AND METHODS}

\section{Liquid crystal materials}

The bent-shaped dimer 1,7-bis[6-(4-hexyloxybenzoyloxy)naphthalene-2-yl]heptane (BNA-76) is prepared in $74 \%$ yield, following a synthetic route described earlier [26].

The rodlike nematogen $4^{\prime}$-cyano[1, 1'-biphenyl]-4-yl 4-hexylbenzoate (6-PEPP- $N$ ) is commercially available (Xi' an Ruilian, China) and is used directly as supplied.

The binary mixture BP12 used in the present study is prepared by dissolving the appropriate quantities of BNA76 and 6-PEPP- $N$ in chloroform. The solvent is evaporated, and the remaining solid is heated above the clearing point. The phase transition temperatures and enthalpies of both compounds and the BP12 mixture are presented in Table I. They are measured on cooling with a Perkin-Elmer DSC differential scanning calorimeter operated at a scanning rate of $5^{\circ} \mathrm{C} / \mathrm{min}^{-1}$ (see Fig. 14 for the DSC scan of the BP12 mixture).

\section{X-ray scattering}

$\mathrm{X}$-ray scattering patterns of aligned samples are recorded with an already described apparatus [80]. Briefly, the $\mathrm{x}$ rays $\left(\lambda_{\mathrm{CuK} \alpha}=1.541 \AA\right)$ produced by a copper fixed tube are monochromatized and point-focused by a doubly curved pyrolytic graphite monochromator and a 0.5 -mm-diameter collimator. The sample is filled into a 1-mm-diameter Lindemann glass capillary (WJM-Glas Müller $\mathrm{GmbH}$, Germany) which is flame-sealed. The capillary is placed in an oven, with $\pm 0.5 \mathrm{~K}$ temperature stability, which is 
TABLE I. Phase transition temperatures in ${ }^{\circ} \mathrm{C}$, enthalpies in $\mathrm{kJ} \mathrm{mol}^{-1}$, and dimensionless value of $\Delta S / R$ (in square brackets) of BNA-76, 6-PEPP- $N$, and the binary mixture BP12.

\begin{tabular}{|c|c|c|c|c|c|c|c|c|c|}
\hline Material & $\mathrm{Cr}$ & & $M_{X}$ & & $N_{\mathrm{TB}}$ & & $N$ & & Iso \\
\hline 6-РЕРP- $N$ & - & $5019.20[7.15]$ & & & & & - & $2270.56[0.13]$ & - \\
\hline BNA-76 & - & $7432.69[11.33]$ & - & $811.29[0.44]$ & • & $1220.15[0.05]$ & - & $1540.68[0.19]$ & - \\
\hline BP12 & - & $4119.91[7.62]$ & - & $1002.44[0.78]$ & - & $1100.10[0.03]$ & $\bullet$ & $1630.62[0.17]$ & \\
\hline
\end{tabular}

calibrated with test substances. The oven lies between the poles of a $1.7 \mathrm{~T}$ electromagnet, sitting at the center of an evacuated camera. The scattering pattern is recorded on an image plate which is read by a Molecular Dynamics scanner. The sample-to-detection distance is usually $60 \mathrm{~mm}$, but a camera extension provides a $120 \mathrm{~mm}$ distance, which gives us access to smaller scattering angles. In terms of scattering vector modulus $q=(4 \pi \sin \theta) / \lambda$, where $2 \theta$ is the scattering angle, the whole accessible $q$ range is $0.1-1.8 \AA^{-1}$, and the instrumental resolution corresponds to $\Delta q=0.023 \AA^{-1}$. From the patterns, various scattered intensity profiles can be extracted, for example, along the director (i.e., $q_{\|}$) or at fixed $q$, through the wide-angle diffuse ring, versus the azimuthal angle.

Unaligned samples (without an applied field) are also studied using a higher-resolution laboratory setup based on a copper rotating anode generator (RU-200BEH, Rigaku Ltd., Japan). The x-ray beam is filtered and focused by a confocal system consisting of two perpendicular graded multilayer mirrors (CMF-12-38Cu6 from Osmic Inc., USA). The samples are contained in glass capillaries as above and mounted in an LTS350 hot stage (Linkam Scientific Instruments, Waterfield, United Kingdom). The scattering signal is measured by a CCD detector cooled down to $30^{\circ} \mathrm{C}$ (Photonic Science, United Kingdom). We use $4 \times 4$ binning, resulting in an effective pixel size of $96 \mu \mathrm{m}$. The sample-to-detector distance is $102.5 \mathrm{~mm}$, yielding an accessible scattering vector range of $0.1-1.9 \AA^{-1}$. The images are integrated, corrected, and calibrated using the Nika suite [81] (version 1.74) running in Igor Pro 7.08.

\section{Electro-optic experiment}

For the electric and electro-optic experiments, we use sandwich-type cells with ITO transparent electrodes deposited on the inner faces of the cell glass plates to apply an electric field along the cell normal. The electrodes are

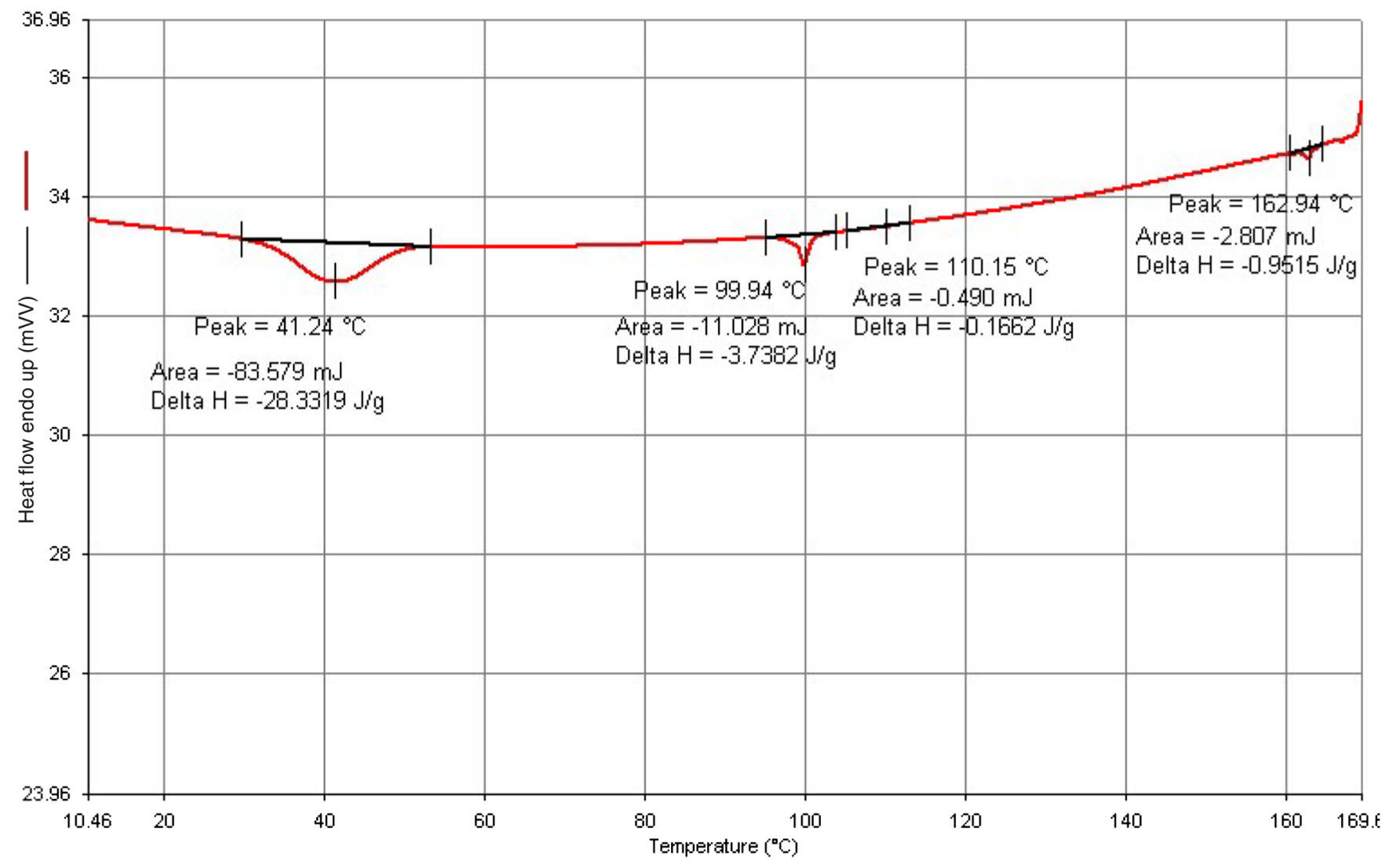

FIG. 14. DSC scan on cooling of the BP12 mixture. 
covered with rubbed polymer alignment layers providing homogeneous planar alignment of the nematic director $\mathbf{n}$ parallel to the rubbing direction $\mathbf{r}$. These cells with different gaps $d$ are either of commercial origin (Instec, USA, $d \approx 5 \mu \mathrm{m}$, and MUT, Poland, $d \approx 10 \mu \mathrm{m}$ ) or prepared in our laboratories $(d \approx 1.4 \mu \mathrm{m})$. The cells are filled by capillarity with the liquid crystal in the isotropic phase and then cooled to the nematic, $N_{\mathrm{TB}}$, and $M_{X}$ phases for the measurements. The temperature of the cell, placed on a heating stage (HS82, Mettler), is controlled with $10 \mathrm{mK}$ accuracy using a homemade temperature controller.

All the polarized-light optical microscopy (POM) observations and measurements are made with a Leitz Ortholux microscope equipped with a digital camera (Dino-lite Pro AM423X). For precise quantitative measurements, we use a highly sensitive photodetector system mounted on the microscope. The latter consists of a photomultiplier tube (PMT) and an optical system which allows for the precise measurement of the intensity of the transmitted light in a small rectangular window in the image plane. For each experiment, we adapt the size of the window to that of the well-aligned single domain under study. The transmitted intensity is measured by the voltage drop $U_{L}$ of the PMT anode current on a load resistance $R_{L}$. The value of $R_{L}$ is chosen high $(1 \mathrm{M} \Omega)$ for experiments with slow dynamics, $\tau>1 \mathrm{~ms}$, to ensure high sensitivity of the measurements. When fast system response is required, $\tau \ll 1 \mathrm{~ms}$, we use $R_{L}=1 \mathrm{k} \Omega$, which provides a response time of approximately $0.4 \mu s$. The amplitude and the time dependence of $U_{L}$ are measured with a digital oscilloscope (DSO-X 2004A, Agilent) which allows averaging the signal over up to 64000 acquisitions and, thus, significantly improving the signal-to-noise $(\mathrm{S} / \mathrm{N})$ ratio. (To avoid any drift and oscillations of the incident light intensity, we use a stabilized voltage source for the microscope lamp.)

The signal applied to the cell electrodes is adapted to the mesophase properties and the kind of electric-field effect under study. The dynamics of the FrTr is investigated by applying square direct current (dc) voltage pulses or square envelope bursts of a sinusoidal alternating current (ac) with a frequency in the range 10-100 kHz. The pulse or burst duration is varied in the range $100 \mu \mathrm{s}-1 \mathrm{~s}$, depending on the response time of the investigated effect. Additionally, for the preparation of homogeneous planar or quasihomeotropic domains, an ac field with variable frequency and amplitude is applied for up to several hours.

The electric signal is produced by an arbitrary waveform generator (TGA12101, TTI) with an amplitude $U \leq 10 \mathrm{~V}$ and is then amplified by a wideband amplifier (Krohn-Hite $7402 \mathrm{M}$ ) up to an amplitude of $U \leq 400 \mathrm{~V}$. To avoid cell deterioration and short circuits, the voltage is limited, depending on the cell gap, to produce a rms field in the cell, $E_{\text {rms }}$, lower than $20 \mathrm{~V} / \mu \mathrm{m}$.

\section{Birefringence measurements}

All the birefringence measurements are performed with uniform single domains prepared by temperatureoscillation annealing of the sample, as described in Appendix C. Depending on the experimental conditions, we measure the cell phase shift $\Delta L$ using different techniques.

For qualitative estimation of the birefringence $\Delta n$, we use a Berek tilting compensator (Olympus). This classical technique [82] is direct and fast, but it is not precise enough for our purposes because of the typical error bars of $10 \mathrm{~nm}$ on $\Delta L$. (This poor precision comes from the low sensitivity of the human eye in detecting the minimum of the transmitted light intensity at the compensation.) Therefore, we use this technique to check the sign of $\Delta L$ and to calibrate the Sénarmont data when the measured phase shift is larger than $\lambda$ (as the Sénarmont technique gives $\Delta L$, modulo $\lambda$ ).

For precise $\Delta L$ measurements, we use a Sénarmont compensator [82] (Leitz). In this case, $\Delta L$ is derived from the value of the analyzer decrossing angle $\alpha_{0}$ that minimizes the transmitted intensity $I(\alpha)$ of the incident monochromatic light $(\lambda=546 \mathrm{~nm})$. To improve the precision, $I(\alpha)$ is measured using the PMT with $R_{L}=1 \mathrm{M} \Omega$, integrating over the area of the single domain. In this way, an excellent $\mathrm{S} / \mathrm{N}$ ratio is obtained, and it is even further improved by averaging the signal over up to several hundreds of oscilloscope acquisitions. For static experiments, e.g., to measure $\Delta L(T)$ at $U=0$ or $\Delta L(U)$ at fixed temperature $T$ and continuously applied voltage $U$, we further improve the precision by measuring $I(\alpha)$ for several $\alpha$ values around the minimum and obtaining $\alpha_{0}$ from a fit of the $I(\alpha)$ curve with a parabola. With this "parabola-fit" technique, the precision is better than $0.1 \mathrm{~nm}$.

For dynamic experiments, i.e., to measure the time evolution of $\Delta L$ when the sample is submitted to square voltage pulses or bursts, we use a small $(1 \mathrm{k} \Omega)$ load resistor for the PMT, providing a response time of the setup much smaller than the response times of our cells. In this case, we rotate the analyzer at $45^{\circ}$ with respect to the transmission minimum, $\alpha=\alpha_{0}-\pi / 4$. In this geometry and for small values of $\Delta L(t)$, the transmitted intensity is approximately [82]

$$
I(t)=\frac{1}{2} I_{0}+\frac{1}{2} I_{0} \sin \left(2 \pi \frac{\Delta L(t)}{\lambda}\right) \approx \frac{1}{2} I_{0}+I_{0} \pi \frac{\Delta L(t)}{\lambda},
$$

where $I_{0}$ is the intensity of the incident light. The PMT signal is measured in real time during each pulse or burst, amplified, and accumulated in the memory of the digital oscilloscope. Averaging the results over a sufficiently large number of acquisitions, we measure $\Delta L(t)$ with a precision better than $0.01 \mathrm{~nm}$ and a time resolution better than $1 \mu s$. 
The techniques described above require a uniform single domain in the PMT window. In the $N_{\mathrm{TB}}-M_{X}$ biphasic coexistence range, this condition is difficult to satisfy, and we often use an "image mapping" technique to measure $\Delta L$. With the Sénarmont compensator, multiple images of the sample are acquired at various values of the analyzer angle $\alpha$ but at constant sample orientation and incident light intensity. Then, the variation with $\alpha$ of the light level recorded in each pixel (or group of pixels) is obtained by computer treatment of the images. Each $I(\alpha)$ curve is fitted with a parabola, giving a map of the angle $\alpha_{0}$ that minimizes $I(\alpha)$, and a map of $\Delta L$. Note that, in the present study, this technique is used to analyze a texture consisting of small single domains of the two $N_{\mathrm{TB}}$ and $M_{X}$ phases, which have lowest light transmission at quite different values of $\alpha$. The large contrast between the two phases and the light scattering by the domain walls increase the noise so that we could use this technique only by averaging over relatively large surfaces (a few hundreds of pixels).

\section{Dielectric measurements}

For dielectric measurements, we use $10-\mu$ m-thick commercial cells treated for planar alignment (MUT, Poland). The active area of the cell, which is submitted to the field, shows perfect planar alignment in the $N$ phase. To obtain good planar alignment over the whole active area in the $N_{\text {TB }}$ phase and, subsequently, in the $M_{X}$ phase, we use the annealing technique described in Appendix C. No dielectric measurements are performed in the biphasic region due to the intrinsic impossibility of obtaining a single domain. Finally, when needed, quasihomeotropic orientation of the whole active area is obtained by slow cooling of the sample under $70 \mathrm{~V}$ ac voltage $(10 \mathrm{kHz})$.

In all cases, the dielectric constants are obtained from the voltage dependence of the cell capacity, $C(U)$, measured in a single domain. To measure $C(U)$, we develop an original setup and an experimental procedure that will be described in more detail elsewhere. In brief, the cell is connected in a passive $R C$ circuit in series with a variable measurement resistor $R_{m}$. An ac voltage with variable frequency $f$ and rms voltage $U_{g}<7 \mathrm{~V}$ is generated using a NI PCIe-6251 data acquisition card from National Instruments, then amplified using a wideband amplifier (Krohn-Hite $7402 \mathrm{M}$ ) up to a much larger rms value, $U_{a}<280 \mathrm{~V}$, and applied to the circuit. The voltage drop $U_{m}$ on the measurement resistor and its phase shift $\delta_{m}$ with respect to $U_{a}$ are measured using the same DAQ card and a suitable voltage divider. The measurement process is controlled by a dedicated proprietary software which provides full control of the voltage range, voltage steps, duration, and time sequence of the data acquisitions. The analysis of the $U_{m}$ and $\delta_{m}$ curves, measured as a function of $U_{a}$ and $f$, and their comparison with the theoretical model of the equivalent circuit of the setup provides the $C(U)$ values.

\section{APPENDIX B: ADDITIONAL X-RAY SCATTERING RESULTS}

The diffractogram of the crystalline phase (black curve) shows, at wide angles, diffraction lines that are most probably broadened by the small size of the crystallites. In contrast, the diffractograms of the $M_{X}, N_{\mathrm{TB}}$, and $N$ phases show no diffraction lines at wide angles, which demonstrates their fluid nature. In addition, three sharp diffraction lines are also visible at small angles for the crystalline phase. The line at $q=0.15 \AA^{-1}$ corresponds to a lattice spacing of $42 \AA$ and is, therefore, related to the average length of the mixture components. The diffractogram of the $M_{X}$ phase (blue curves) shows only a single sharp reflection, at $q=0.28 \AA^{-1}$, so that the phase must have an interdigitated structure (since optical measurements rule out any director tilt; see the main text). The diffractograms of both the $N_{\text {TB }}$ and $N$ phases (green and red traces, respectively) show no sharp diffraction lines at all, as expected for these mesophases that lack any kind of long-range positional order of the molecules.

Azimuthal profiles of the scattered intensity, at fixed scattering vector modulus at the maximum of the wideangle diffuse ring, are extracted from the scattering patterns shown in Fig. 15. Surprisingly, the width of these profiles
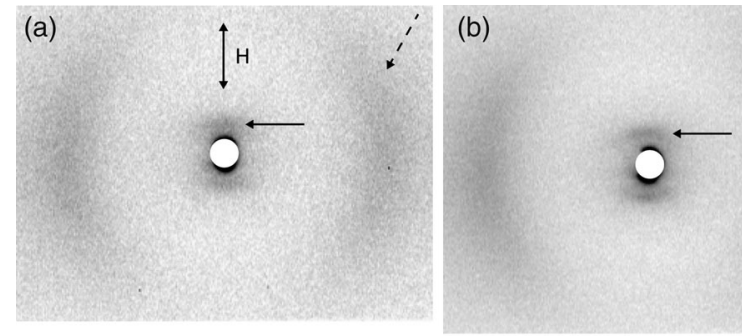

(c)
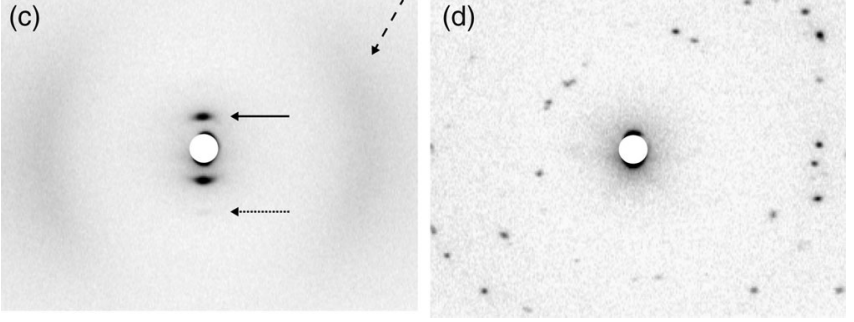

FIG. 15. X-ray scattering patterns of a BP12 sample aligned by a $1.7 \mathrm{~T}$ magnetic field $H$ [double-headed arrow in (a)] recorded with a $60 \mathrm{~mm}$ sample-to-detection distance. (a) In the $N$ phase ( $\left.T=117^{\circ} \mathrm{C}\right)$; (b) in the $N_{\mathrm{TB}}$ phase $\left(T=107^{\circ} \mathrm{C}\right)$; (c) in the $M_{X}$ phase $\left(T=97^{\circ} \mathrm{C}\right)$; and (d) in the crystalline phase (room temperature). White disks at the pattern centers represent the beam stop. In (a)-(c), the dashed arrow points to the wide-angle diffuse ring. In (a) and (b), the solid arrow points to small-angle diffuse streaks. In (c), the solid arrow points to one of the two first-order smectic reflections, while the dotted arrow points to one of the two second-order smectic reflections. In (d), the pattern shows only sharp Bragg reflections arising from several large crystallites in reflection position in the x-ray beam. 


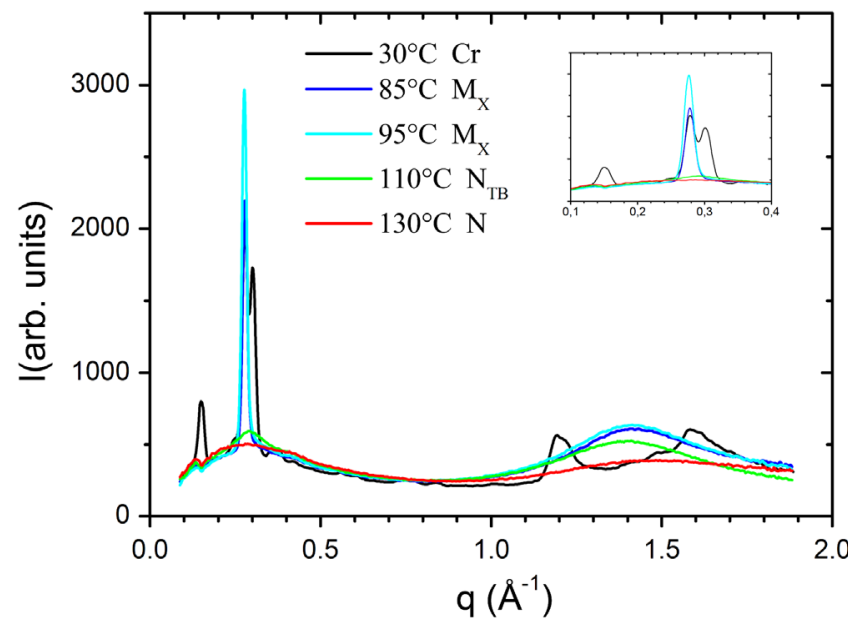

FIG. 16. Powder x-ray diffractograms of a BP12 sample in the crystalline, $M_{X}, N_{\mathrm{TB}}$, and $N$ phases. The inset shows a magnification of the low-angle region.

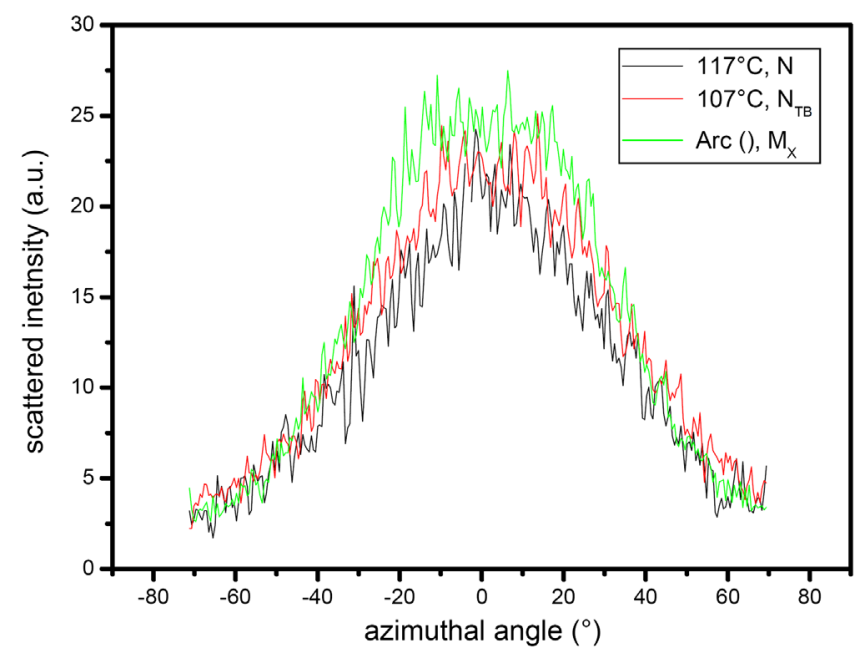

FIG. 17. Azimuthal profiles of the scattered x-ray intensity of a BP12 sample aligned in a magnetic field in the $N, N_{\mathrm{TB}}$, and $M_{X}$ mesophases.

increases with decreasing temperature, in particular, in the $M_{X}$ phase. This unusual feature suggests that the mesogenic cores are actually tilted with respect to the normal to the smectic layers, as in $\mathrm{SmC}$ - and $\mathrm{SmC}_{A^{-}}$type phases.

\section{APPENDIX C: PREPARATION OF LARGE UNIFORM DOMAINS FOR THE MEASUREMENTS}

\section{Growth of planar single domains of the $N_{\mathrm{TB}}$ and $M_{X}$ phases}

To measure $\Delta L$, we need large enough single domains with uniform planar or homeotropic alignment. In the $N$ phase, excellent planar orientation (with a small uniform pretilt of approximately $2^{\circ}$ ) of the whole sample is induced

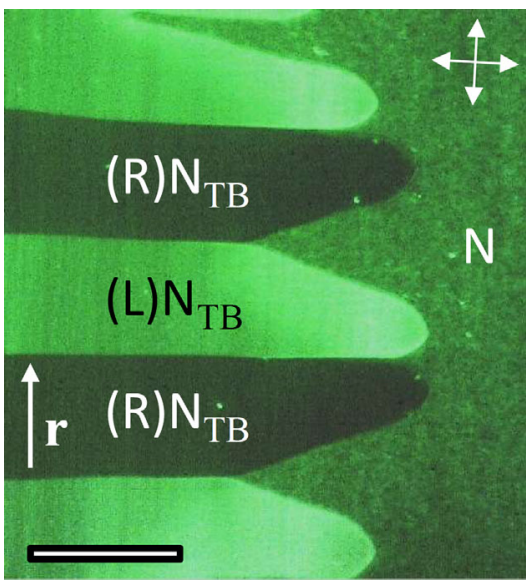

FIG. 18. Growth of $N_{\mathrm{TB}}$ monochiral domains (on the left side) in the $N$ phase (on the right side) at the $N-N_{\mathrm{TB}}$ transition of BP12 ( $\left.T=108.9^{\circ} \mathrm{C}, d=9.8 \mu \mathrm{m}\right)$. To evidence the alternating sign of the chirality of the domains, they are observed in monochromatic light, $\lambda=546 \mathrm{~nm}$, between crossed polarizers (white doubleheaded arrows) rotated at $3^{\circ}$ with respect to the rubbing direction r. (Scale bar $100 \mu \mathrm{m}$.)

by the alignment layers. Slowly cooling the cell through the $N-N_{\mathrm{TB}}$ transition, in a small horizontal thermal gradient, produces large $N_{\mathrm{TB}}$ monochiral domains, with uniform planar orientation of the helix axis $\mathbf{h}$ oriented as the director in the previous $N$ texture (Fig. 18). Close to the transition, these domains are perfectly uniform, with $\mathbf{h} \| \mathbf{r}$, and monochiral, with the sign of the chirality alternating from one domain to the next (Fig. 19). On further cooling, however, we observe the usual stripe instabilities [19] of the $N_{\text {TB }}$ phase [Fig. 20(a)]. This result hinders precise birefringence measurements and prevents growing large single domains of the $M_{X}$ phase on further cooling.

To solve this issue, we impose temperature oscillations, with a typical amplitude of $\pm 1{ }^{\circ} \mathrm{C}$ and $1-3$ min period while cooling the sample. This temperature cycling, together with periodic application of weak electric fields (approximately $0.5 \mathrm{~V} / \mu \mathrm{m}$ ), allows us to keep the monochiral $N_{\mathrm{TB}}$ domains uniform [Fig. 20(d)]. Finally, starting from well-annealed and uniform $N_{\mathrm{TB}}$ single domains and slowly (approximately $0.1^{\circ} \mathrm{C} / \mathrm{min}$ ) decreasing the temperature, we achieve the growth of the required highly uniform single domains of the $M_{X}$ phase (Fig. 4). As the temperature is slowly decreased, the $M_{X}$ single domains grow, in a reversible way, until the whole sample turns into the $M_{X}$ phase. At constant temperature, the coexisting $M_{X}$ and $N_{\mathrm{TB}}$ regions remain at equilibrium (Fig. 21).

However, the azimuthal orientation of the slow axis $\mathbf{N}$ of the $M_{X}$ domains is still not perfect. When the sample is observed with the rubbing direction $\mathbf{r}$ parallel to one of the crossed polarizers, the texture observed is smecticlike, consisting of subdomains with perfectly uniform azimuthal orientation, separated by sharp grain boundaries. Rotating the sample by a small angle, between crossed polarizers, 

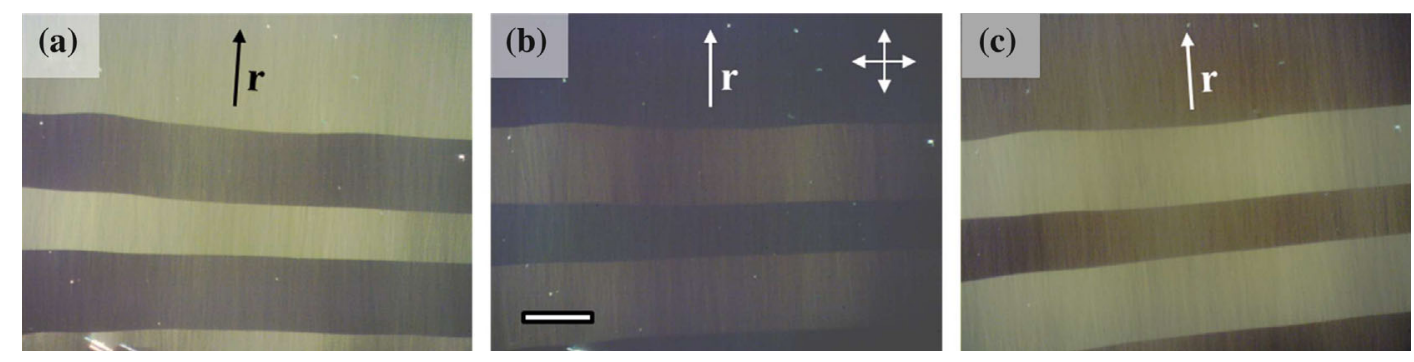

FIG. 19. Alternating monochiral $N_{\mathrm{TB}}$ domains of BP12 observed between crossed polarizers $\left(T=108.8^{\circ} \mathrm{C}, d=9.8 \mu \mathrm{m}\right)$. In both kinds of domains, the optic axis $\mathbf{N}$ is parallel to the rubbing direction $\mathbf{r}$. When $\mathbf{r}$ is parallel to the input polarizer (b), both kinds of domains appear dark. When the sample is rotated by $\pm 4^{\circ}[(\mathrm{a})$ and (c)], a strong transmittance contrast appears between the two kinds of domains. The inversion of this contrast with the sign of the rotation indicates the different signs of the chirality of adjacent domains. (Scale bar $100 \mu \mathrm{m}$.)
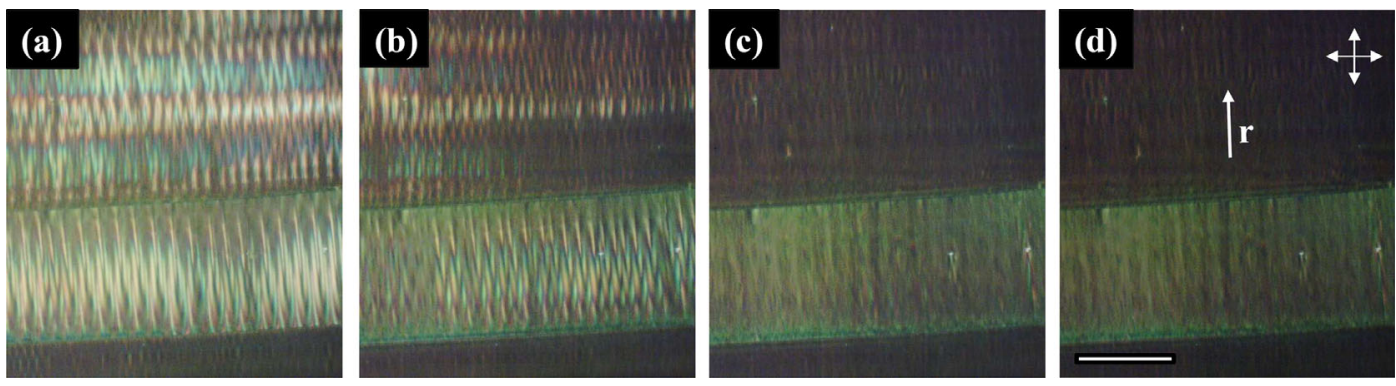

FIG. 20. Stripe instabilities in the monochiral $N_{\mathrm{TB}}$ domains of BP12 (a) and their annealing after thermal cycling and repeated application of a weak electric field $\left(T=108.0^{\circ} \mathrm{C}, d=9.8 \mu \mathrm{m}\right)$. After annealing (b)-(d), the domains are again uniform and their optic axis $\mathbf{N}$ is parallel to the rubbing direction $\mathbf{r}$. The weak residual heterogeneities after annealing (d) are due to the surface memory of the alignment layers. (Scale bar $100 \mu \mathrm{m}$.)

reveals that $\mathbf{N}$ deviates from $\mathbf{r}$ by a few degrees in these subdomains, typically less than $\pm 3^{\circ}$ [Figs. 22(a)-22(c)]. Note that these small deviations do not significantly affect the precision of the birefringence measurements.

As described above, the birefringence experiment requires single domains of area typically larger than $20 \mu \mathrm{m} \times 20 \mu \mathrm{m}$ and uniform planar alignment, both
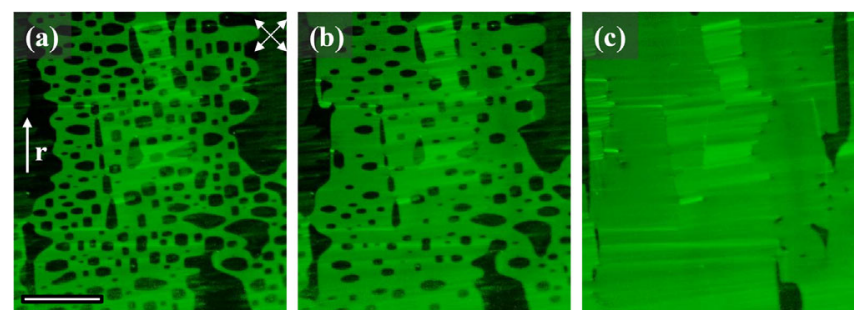

FIG. 21. Biphasic coexistence of the $M_{X}$ (bright) and $N_{\mathrm{TB}}$ (dark) phases in a $d=1.4 \mu \mathrm{m}$ planar-alignment cell at different temperatures: $100{ }^{\circ} \mathrm{C}(\mathrm{a}), 98^{\circ} \mathrm{C}(\mathrm{b})$, and $97^{\circ} \mathrm{C}(\mathrm{c})$. To visualize the different birefringence of the two phases, the cell is observed under polarized monochromatic light $(\lambda=546 \mathrm{~nm})$ with a Sénarmont compensator and with an analyzer rotated by an angle optimizing the contrast between the two phases. Each image is recorded after $15 \mathrm{~min}$ of relaxation at fixed temperature to reach equilibrium between the two phases. (Scale bar $100 \mu \mathrm{m}$.) zenithal and azimuthal, of the slow axis $\mathbf{N}$. The requirements for the dielectric experiment are in a way more stringent, because it averages the signal arising from the whole electrode-covered area of the cell. In that case, we need good planar alignment over this square active area of typically $5 \mathrm{~mm}$ size. Fortunately, the azimuthal uniformity of the alignment is not so relevant for dielectric measurements, because they are not sensitive to in-plane rotation of the directors. Therefore, we align the whole active area of the cell in the $N_{\mathrm{TB}}$ and $M_{X}$ phases using the same annealing treatment described above. In contrast, a large enough single domain is impossible to produce at the $N_{\mathrm{TB}}-M_{X}$ coexistence, which prevents any dielectric measurements in the biphasic coexistence range.

\section{Growth of quasihomeotropic domains of the three phases}

To prove directly the biaxiality of the $M_{X}$ phase, we need a homeotropically aligned sample, i.e., with primary director $\mathbf{n}$ oriented parallel to the cell normal and to the observation direction. The same orientation is also needed for the measurement of the complete set of the dielectric tensor eigenvalues in the different mesophases. Unfortunately, it is notoriously difficult to achieve the homeotropic alignment of bent-shaped dimers by surface 

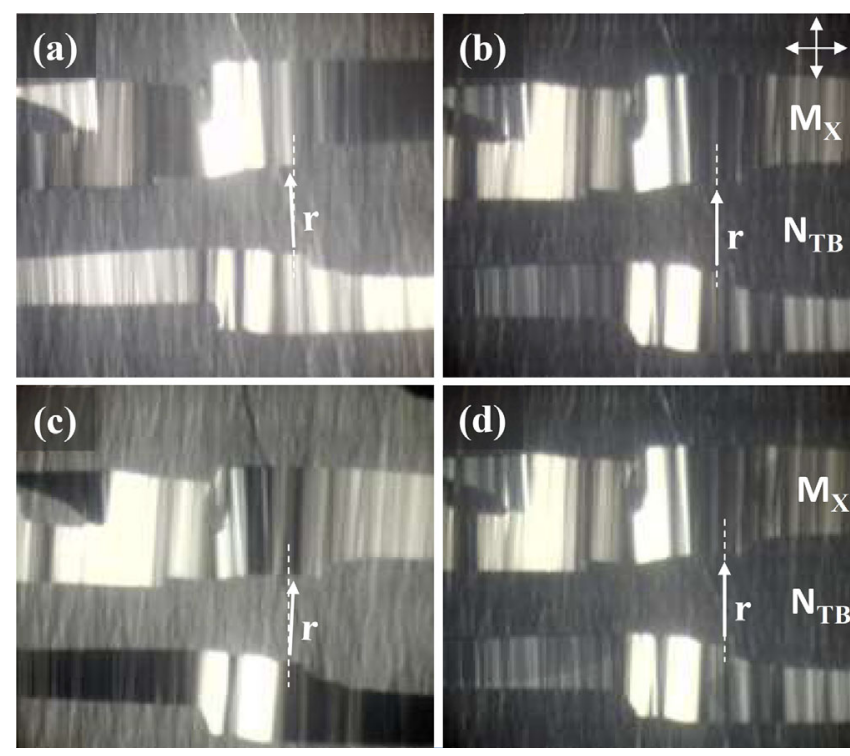

FIG. 22. Azimuthal alignment of the $M_{X}$ domains coexisting with the $N_{\text {TB }}$ phase at $101.8^{\circ} \mathrm{C}$. The sample is observed between crossed polarizers and rotated at angle $\psi=-2^{\circ}, 0^{\circ},+2^{\circ}$, and $0^{\circ}$ in (a), (b), (c), and (d), respectively. Here, $\psi$ is the angle between the rubbing direction $\mathbf{r}$ and the input polarizer. The slow axis $\mathbf{N}$ of the $M_{X}$ regions is approximately parallel to $\mathbf{r}$. The azimuthal deviations of $\mathbf{N}$ from $\mathbf{r}$, at angles typically smaller than $\pm 3^{\circ}$, are due to slight disorientations of the smectic single domains of the $M_{X}$ phase. The analysis of the transmitted light and the smectic textures shows that $\mathbf{N}$ is vertical in the pictures, perpendicular to the smectic layers that are horizontal, which confirms the $\operatorname{Sm} A$ (and not $\mathrm{SmC}$ ) nature of the $M_{X}$ phase. In (d), an electric field, larger than the threshold of the Freédericksz-like transition, is applied to the sample $\left(f=6 \mathrm{kHz}, U_{\mathrm{rms}}=7 \mathrm{~V}\right)$.

treatments in any of the $N$ and $N_{\text {Тв }}$ phases. We try several classic surface treatments that usually provide homeotropic alignment for most of the nematic and smectic $A$-forming compounds: grafted silane surfactant layers, polyimide layers developed for homeotropic alignment, and even bare glass or ITO surfaces. Without surprise, we also fail to obtain a homeotropic alignment of either BP12 or other mixtures of BNA-76 in any phase. In all these attempts, we obtain in the nematic phase a poor, very inhomogeneous planar alignment with azimuthal orientation varying at random on the two cell surfaces and dominated by the anchoring memory. In the $N_{\mathrm{TB}}$ and $M_{X}$ phases, this memorized alignment (incompatible with the pseudolayered or layered structure) turns in a fan-shaped texture, which is unsuitable for our experiments.

However, we produce a quasihomeotropic alignment in our cells treated for planar alignment by taking advantage of the positive dielectric anisotropy of BP12, $\Delta \varepsilon \approx 2$. We apply continuously a strong ac electric field $\left(E_{\mathrm{rms}}=17 \mathrm{~V} / \mu \mathrm{m}, f=10 \mathrm{kHz}\right)$ throughout the thermal history of the sample. In the nematic phase, this application yields a perfect homeotropic alignment of the bulk of the cell, leaving only two thin (approximately $10-\mathrm{nm}$-thick) surface layers with strong director distortion, which is due to the competition of the aligning torques of the surface and the field. The resulting texture is quasihomeotropic, with a small residual phase shift of about $3 \mathrm{~nm}$ and slow axis parallel to $\mathbf{r}$. When the sample is cooled slowly under the field to the $N_{\text {TB }}$ phase, this quasihomeotropic texture is preserved, with some decrease of the residual birefringence. In the bulk of the sample, the helix axis is homeotropic, and the $N_{\mathrm{TB}}$ pseudolayers are parallel to the surfaces, like the layers of a homeotropically aligned smectic phase. Finally, cooling further the sample under the field, we observe the growth of large and uniform quasihomeotropic domains of the $M_{X}$ phase (Fig. 5). These domains are birefringent, which directly confirms that the $M_{X}$ phase is biaxial.

Although the growth of quasihomeotropic monodomains is possible only under a strong electric field, the orientation of the layers parallel to the cell surface is kept when the field is removed at a low enough temperature $\left(T<85^{\circ} \mathrm{C}\right)$. Because of the "frozen" layer structure, the $\mathbf{n}$ director remains homeotropic, while $\mathbf{m}$ is parallel to the surface. In this case (Fig. 23), we observe texture instabilities with characteristic zebra patterns between crossed polarizers, due to the very weak (or nonexistent) anchoring of $\mathbf{m}$ parallel to $\mathbf{r}$. In the zebra pattern, $\mathbf{m}$ spontaneously rotates by a few quarter turns in the surface plane. The transmitted intensity varies in the field of view as $\sin ^{2} 2 \varphi$, where $\varphi$ is the angle between $\mathbf{m}$ and one of the crossed polarizers. This variation again confirms the lack of revolution symmetry around $\mathbf{n}$ and, therefore, the biaxiality of the $M_{X}$ phase.

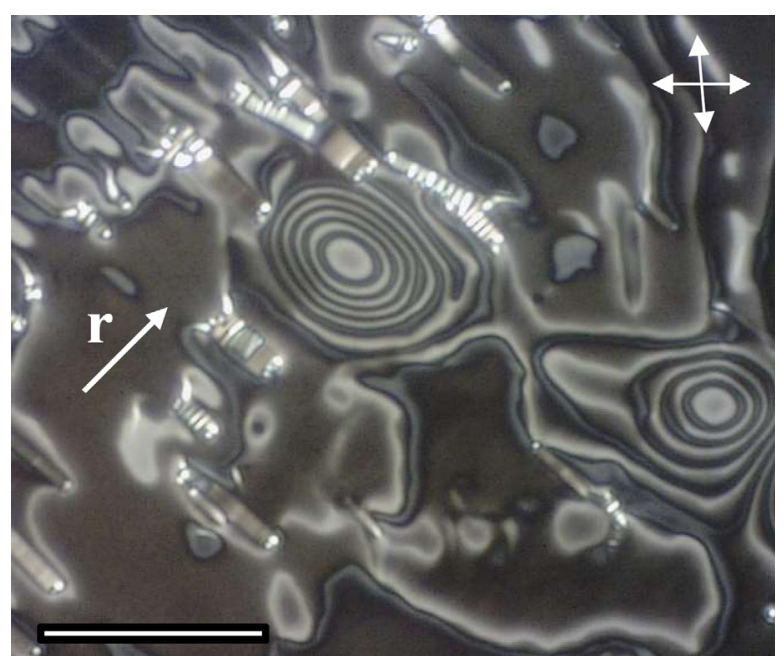

FIG. 23. Homeotropically aligned $M_{X}$ phase after field removal ( $d=1.4 \mu \mathrm{m}, T=85^{\circ} \mathrm{C}$ ). The smectic layers are parallel to the image plane, $\mathbf{n}$ is perpendicular to it, and $\mathbf{m}$ is parallel to the layers, in the image plane. The sample is birefringent, with $\Delta n \sim 0.02$, and the slow axis is parallel to $\mathbf{m}$. In the striped areas, $\mathbf{m}$ rotates in the plane, and each band corresponds to a $90^{\circ}$ rotation. (Scale bar $50 \mu \mathrm{m}$.) 


\section{APPENDIX D: INTERPRETATION OF THE BIREFRINGENCE DATA}

In the absence of a field, the birefringence measured in the different phases of BP12 depends on the values of the components of the order parameter tensor $\mathbf{Q}$, on its surfaceimposed orientation in the single domains under study, and on the structure of the phase (i.e., homogeneous or heliconical). In principle, all of these parameters are sensitive to applied fields, leading to variation of the birefringence. However, for realistically strong fields, such as those applied in our experiment, only the reorientation of $\mathbf{Q}$ is significant and should be taken into account.

In the birefringence experiments, the optical path difference $\Delta L$ between the different normal modes of light propagating in the anisotropic medium is measured. In our experimental geometry, the rubbing axis $\mathbf{r}$ lies in the cell plane, oriented at $45^{\circ}$ with respect to the polarizer, and monochromatic light propagates along the cell normal. Without a field, the optical properties of all three phases $(N$, $N_{\mathrm{TB}}$, and $M_{X}$ ) are uniform, with the major axis of the ellipsoid of indices, $\mathbf{N}$, oriented parallel to $\mathbf{r}$. Note that, despite the strong nematic director distortion in the $N_{\mathrm{TB}}$ phase, it is optically uniaxial with $\mathbf{N}$ parallel to the helix axis, $\mathbf{h}(\mathbf{N}\|\mathbf{h}\| \mathbf{r})$ [54]. Therefore, without a field, we have $\Delta L=\left(n_{\|}-n_{\perp}\right) d$ in the uniaxial $N$ and $N_{\text {TB }}$ phases, where the subscripts of the refraction indices refer to the polarization direction with respect to $\mathbf{N}$. In the biaxial $M_{X}$ phase, in the absence of a field, we have $\Delta L=\left(n_{n n}-n_{m m}\right) d$, where the subscripts of the refraction indices now refer to the polarization direction parallel to, respectively, either $\mathbf{n}$ or $\mathbf{m}$. Similarly, under an infinitely strong field $(U=\infty)$, all three phases are again uniform, but with the major axis of the ellipsoid of indices oriented along the cell normal. Therefore, $\Delta L=0$ in the uniaxial phases and $\Delta L=$ $\left(n_{m m}-n_{k k}\right) d$ in the biaxial $M_{X}$ phase.

When a sufficiently large but finite voltage is applied to the $N$ or $M_{X}$ phase, the cell undergoes a FrTr and the orientation of $\mathbf{N}$ is no longer uniform but varies along the $z$ axis. (We do not consider here the more complex $N_{\mathrm{TB}}$ case, where the reorientation of $\mathbf{h}$, and, therefore, of $\mathbf{N}$, is a nonequilibrium defect-mediated process.) In the $N$ case, $\mathbf{n}$ rotates away from $\mathbf{r}$ but still remains in the same zenithal plane. In the $M_{X}$ case, $\mathbf{n}$ remains orthogonal to the rigid smectic layers, but $\mathbf{m}$ tilts away from its initial orientation while remaining in the same zenithal plane. Because the distortion of the respective director is planar (without twist) in both cases, there is no rotation of the polarization plane of light during its propagation in the cell, and the phase shift in the $N$ phase is

$$
\Delta L(U)=\int_{0}^{d}\left[\frac{n_{\|} n_{\perp}}{\sqrt{n_{\|}^{2} \cos ^{2} \theta+n_{\perp}^{2} \sin ^{2} \theta}}-n_{\perp}\right] d z,
$$

where $\theta=\theta(z, U)$ is the position- and voltage-dependent angle between the field and $\mathbf{n}$ [51].

By analogy, we obtain for the $M_{X}$ case

$\Delta L(U)=\int_{0}^{d}\left[n_{n n}-\frac{n_{m m} n_{k k}}{\sqrt{n_{m m}^{2} \cos ^{2} \theta+n_{k k}^{2} \sin ^{2} \theta}}\right] d z$

where $\theta=\theta(z, U)$ now refers to $\mathbf{m}$.

The field-induced tilt of the director, $\theta(z, U)$, during the FrTr in the $N$ phase, can be obtained as a function of the applied voltage and the material constants (elastic moduli, dielectric tensor components, etc.) by integrating the EulerLagrange equation corresponding to the free energy of Eq. (3) $[58,59,65]$. This classical approach allows one to interpret the $\Delta L(U)$ data acquired in the $N$ phase $[58,59,83]$ and to extract the threshold field of the FrTr, $U_{c}^{n}$, and the nematic birefringence, $\Delta n=n_{\|}-n_{\perp}$. Moreover, based on the similarity of the elastic behaviors of the $N$ and $M_{X}$ phases, we use here the same approach to interpret the $\Delta L(U)$ data acquired during the BFrTr in the $M_{X}$ phase and to extract the threshold field of the BFrTr, $U_{c}^{m}$, and the variation of the refraction index, $n_{m m}-n_{k k}$, related to the reorientation of $\mathbf{m}$.

When the voltage is applied as dc pulses or ac bursts, the director orientation in the cell and, therefore, the optical phase shift become time dependent. For the nematic phase, the reorientation of $\mathbf{n}$ during the FrTr depends on the applied voltage, the cell thickness, the rotational and translational viscosities, and the involved distortion modes [72]. The precise description of the time-dependent optical response of the cell requires a numerical simulation. However, a simple analytical description is possible in two important particular cases for which the time dependence of the tilt angle of $\mathbf{n}$ in the middle of the cell, $\theta_{\min }(t)$, follows a simple exponential law: $\theta_{\min }(t) \sim \exp (-t / \tau)$. Here, $\tau=\tau_{\text {on }}$ and $\tau_{\text {off }}$ stands for the characteristic response time when the field is switched on or off, respectively. This approximate description, well known for the FrTr of the $N$ phase [72], is extended here, by analogy, to the case of the BFrTr for the $M_{X}$ phase.

The first case is that of $\tau_{\text {on }}$ when the applied voltage is much higher than the threshold field $U \gg U_{c} ; \tau_{\text {on }}$ is voltage dependent and is approximately given by

$$
\tau_{\mathrm{on}}^{i} \approx \frac{\gamma_{1}^{i}}{K_{11}^{i}}\left(\frac{d U_{c}^{i}}{\pi U}\right)^{2}
$$

where the superscript $i=n, m$ indicates the director involved in the Fréedericksz transition for the $N$ or $M_{X}$ phase, respectively, and $\gamma_{1}^{i}$ is the rotational viscosity for reorientation of that director. The strong voltage dependence of $\tau_{\text {on }}$ allows achieving very fast on response of nematic devices by simply applying high enough voltage. 
The second simple case is related to the relaxation process upon field removal. Then, multiple relaxation modes are excited, corresponding to different wavelengths, $2 d /(2 \nu+1)$, where $\nu=0,1, \ldots$ is an integer, of the director distortion. The off-relaxation times of the different modes decrease as $1 /(2 \nu+1)^{2}$, but, after a fast initial relaxation, the process is dominated only by the slowest time, corresponding to $\nu=0$ :

$$
\tau_{\mathrm{off}}^{i} \approx \frac{\gamma_{1}^{i}}{K_{11}^{i}}\left(\frac{d}{\pi}\right)^{2}
$$

This time depends only on the cell thickness and not on the applied field.

Note that the on- and off-relaxation times of the director, defined above, are related in a simple way to the optical relaxation times $\tau_{\mathrm{on} / \mathrm{off}}^{\mathrm{opt} i}(i=n, m)$, which are measured in our experiment [see Eqs. (D1) and (D2)]: $\tau_{\mathrm{on} / \mathrm{off}}^{i}=2 \tau_{\mathrm{on} / \mathrm{off}}^{\mathrm{opt}} \cdot$

\section{APPENDIX E: INTERPRETATION OF THE DIELECTRIC DATA}

The cell capacitance $C(U)$ is measured as a function of the voltage applied to the LC layer in the dielectric experiment. Using various surface- and field-induced alignments, all three eigenvalues of the dielectric tensor $\varepsilon$ can be measured in the three mesophases under study. In the uniaxial $N$ and $N_{\mathrm{TB}}$ phases, the components $\varepsilon_{\perp}=\varepsilon_{k k}=$ $\varepsilon_{m m}$ and $\varepsilon_{\|}=\varepsilon_{n n}$ are obtained by extrapolating the $C(U)$ results (measured in a cell with planar surface alignment) to $U=0$ and $U \rightarrow \infty$, respectively. (In these extreme cases, $\mathbf{n}$ is uniform and oriented either perpendicular or parallel to the field, respectively.) In the $M_{X}$ phase, the components $\varepsilon_{k k}$ and $\varepsilon_{m m}$ are obtained by extrapolating the $C(U)$ data, measured in a sample with planar orientation of $\mathbf{n}$, to $U$ values, respectively, much smaller or much higher than the threshold voltage of the BFrTr. Indeed, in these two limit cases, the $\mathbf{Q}$ tensor is oriented with either the $\mathbf{k}$ or $\mathbf{m}$ director parallel to the field. The last component of the

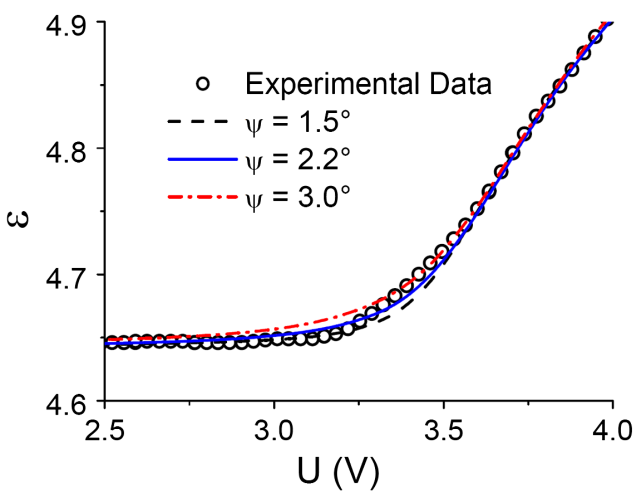

dielectric tensor, $\varepsilon_{n n}$, is obtained from the $C(U)$ data measured in a quasihomeotropic sample, produced by crossing the $N-N_{\mathrm{TB}}-M_{X}$ transitions under a strong field (see Appendix C).

In the $N$ and $M_{X}$ phases, $C(U)$, measured in a planar cell, varies smoothly and reversibly during the FrTr. The wellknown theoretical description of the FrTr in the $N$ phase $[64,65]$ gives $C(U)$ as a function of the elastic, electric, and anchoring properties of the liquid crystal. Thus, the fit of the $C(U)$ data with the theory provides [58-60] the values of $\varepsilon_{\perp}, \varepsilon_{\|}$, and the elastic moduli of the primary director, $K_{11}^{n}$ and $K_{33}^{n}$. Using a proprietary numerical simulation program, we measure precisely these parameters, as well as the pretilt angle $\psi^{n}$, and (approximately) the surface anchoring energy $W_{s}^{n}$ for $\mathbf{n}$ [see Fig. 8(a) for an example of the excellent fit of the data with the theory].

We apply a similar approach for the treatment of the dielectric data of the biaxial $M_{X}$ phase thanks to the analogy of the BFrTr with the usual FrTr in the nematic. The fit of the data with the numerical simulation [Fig. 8(a)] gives precisely the dielectric tensor components $\varepsilon_{k k}$ and $\varepsilon_{m m}$ and the elastic moduli of the secondary director, $K_{11}^{m}$ and $K_{33}^{m}$.

The pretilt angle $\psi^{m}$ and the surface anchoring energy $W_{s}^{m}$ are also estimated from the fit with the theoretical curves, as they are independent fitting parameters. The experimental data are compared in Fig. 24 (left) with the theoretical curves calculated for three different values of $\psi^{m}$ and for the best-fit values of all the other parameters [the region around $U=U_{c}$ is shown only because the curvature of $C(U)$ there is very sensitive to the value of $\left.\psi^{m}\right]$. Because of the symmetry of the unidirectionally rubbed polyimide layers, we expect $\psi^{m}=0$ (contrary to the pretilt of the primary director, $\psi^{n} \neq 0$, because the inversion symmetry of the surface director is broken by the rubbing process). The measured small, but finite, value $\psi^{m}=2.2 \pm 0.7^{\circ}$ is probably due to easy axis gliding [84] under the strong electric torques applied during the experiments. The significant deviation of the experimental curve

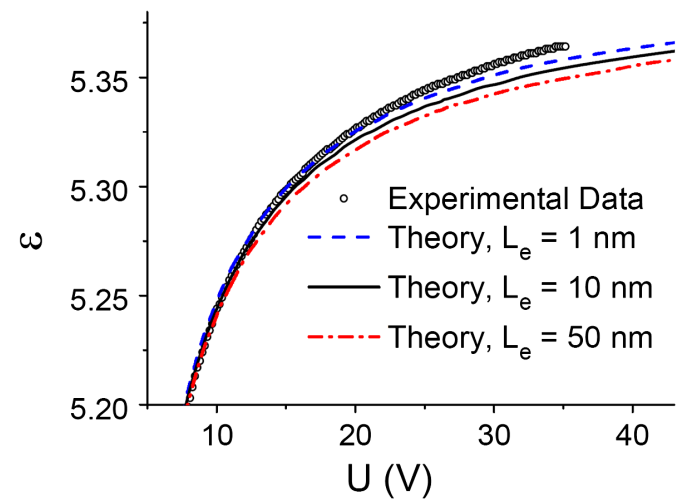

FIG. 24. Estimations of the pretilt angle $\psi^{m}$ (left) and the anchoring extrapolation length $L_{e}^{m}$ (right) for the secondary director in the $M_{X}$ phase $\left(T=95^{\circ} \mathrm{C}\right)$. 
from the theoretical ones indicates some local variations of $\psi^{m}$ on the cell surfaces.

To take into account the finite anchoring energy $W_{s}^{m}$ of the $\mathbf{m}$ director, we use as a fitting parameter the corresponding de Gennes extrapolation length [51] $L_{e}^{m}=$ $K_{11}^{m} / W_{s}^{m}$. Figure 24 (right) shows the deviation of the experimental data from the best-fit theoretical curves calculated with different fixed values of $L_{e}^{m}$ (this parameter influences mainly the high-voltage region). Qualitatively, the large deviation of the data from the $L_{e}^{m}=50 \mathrm{~nm}$ curve indicates that $L_{e}^{m} \ll 50 \mathrm{~nm}$, i.e., that $W_{s}^{m} \gg 0.2 \mathrm{~mJ} / \mathrm{m}$, which is a rather strong value. Surprisingly, the experimental curve is even higher than the curve calculated with infinite anchoring energy. This feature is most probably due to a small field-induced increase of the biaxial order, leading to a higher $\varepsilon_{m m}$ value and to an increase of the cell capacitance $C(U)$.

Another way to estimate $L_{e}^{m}$ is to compare the values of $U_{c}$ measured in cells with significantly different gaps (this approach uses only low- $U$ data, thus avoiding high-field artifacts). In fact, for weak anchoring, the threshold value is decreased by a factor of $\left(1+2 L_{e}^{m} / d\right)$ [85]. The systematic difference between the BFrTr thresholds measured at the same temperature for the $d=1.4 \mu \mathrm{m}$ - and $d=9.8 \mu \mathrm{m}$ thick cells is less than $1 \%$ (it is, in fact, hidden in the statistical noise of about 2\%). This result corresponds to $L_{e}^{m} \approx 10 \mathrm{~nm}$, i.e., again to a very strong anchoring energy $W_{s}^{m} \approx 1 \mathrm{~mJ} / m$.

In contrast to the birefringence measurements, the dielectric technique does not allow for the investigation of the dynamics of the FrTr and BFrTr.

\section{APPENDIX F: IN-LAYER ORIENTATIONAL ORDER OF THE $M_{X}$ PHASE}

The x-ray scattering data clearly show that the $M_{X}$ phase is smectic and that the bent-shaped BNA-76 dimers are completely intercalated in the smectic layers. Indeed, the smectic period $d_{0}$ is about half of the dimer length $L$, $d_{0} \approx L / 2$, which indicates that the smectic layers are not formed by the dimer molecules but by the monomer mesogenic units. Each monomer in a layer belongs to a dimer that spans, with equal probability, over one of the two adjacent layers. (Strictly speaking, this description is valid for the $M_{X}$ phase of the pure BNA-76 compound. In the case of the BP12 mixture that is investigated here, a part of the monomers in each layer is replaced by the 6-PEPP- $N$ molecules, which have approximately the same length as the monomer. For simplicity and because of the small amount of 6-PEPP- $N$, we neglect this detail here.)

Moreover, the intensity distribution in the wide-angle diffuse scattering ring and the relation $d_{0} \approx L / 2$ both suggest that the $M_{X}$ phase is an orthogonal smectic phase of the bent-shaped dimers, i.e., that the (primary) director $\mathbf{n}$ of the phase, defined by the average orientation of the long axes of the dimers, is perpendicular to the smectic layers. This feature is unambiguously confirmed by our electrooptic observations: Without a field, the slow axis of the sample is along the normal to the layers. When the field is applied, the cell birefringence increases, but the slow axis keeps its orientation, which indicates that $\mathbf{n}$ is not reoriented by the field and remains perpendicular to the layers. Moreover, all our optical and electric experiments indicate that the $M_{X}$ phase is biaxial. Therefore, the $M_{X}$ phase of the bent-shaped dimers is an orthogonal biaxial smectic phase. This phase is already reported in the literature for several compounds under different names: $\operatorname{Sm} A_{b}$ [42], $\mathrm{Sm}_{M}$ [51], etc. Here, we use the name $\operatorname{Sm} A_{b}$ for this orthogonal biaxial smectic phase.

However, the orientational order of the monomers in each smectic layer also matters, since their para-axes are tilted with respect to $\mathbf{q}_{0}$, due to the bent shape of the dimers. Depending on the in-layer orientational order of the monomers, different structures of this phase are possible.

One of them, which has been already proposed for the intercalated smectic phase of bent-shaped dimers [9-11,13,32-34,41,44-49], is illustrated in Fig. 3. In this case, the para-axes $\mathbf{p}$ of all the monomers in a layer are tilted in the same direction, resulting in a SmC layer with tilted director $\langle\boldsymbol{p}\rangle$. Because of the bent shape of the dimer molecules, the tilt of $\langle\boldsymbol{p}\rangle$ alternates from one layer to the next, as in an anticlinic smectic $C, \mathrm{Sm}_{A}[4,42,49,50]$. Statistically, due to the intercalation, the monomers of a given layer belong with the same probability to dimers oriented in opposite directions. Therefore, the average polarization of the layer vanishes, and the phase is not ferroelectric despite the similarity of its structure (except for the intercalation) with that of the antiferroelectric $\operatorname{Sm} A_{\mathrm{PA}}$ phase $[14,15]$. Then, the in-layer orientational order of the monomers is described by a biaxial tensor with a tilted primary director. We refer to this kind of intercalated smectic as $\operatorname{Sm} A_{b}\left(\operatorname{Sm} C_{A}\right)$, where we specify the in-layer orientational order of the monomers in brackets.

Another possible structure is sketched in Fig. 25. In that case, the tilt of the monomers in each layer is doubly degenerate, with their para-axes lying in the same plane but tilted (with equal probability) one way or the other. The in-plane order parameter tensor of the monomers is again biaxial, but the primary director $\langle\boldsymbol{p}\rangle$ is perpendicular to the layer. As the in-plane order of the monomers is $\operatorname{Sm} A_{b}$, we refer to this structure as $\operatorname{Sm} A_{b}\left(\operatorname{Sm} A_{b}\right)$. (We note that in this case the monomer layer has the same $D_{2 h}$ symmetry as in the classic $\operatorname{Sm} A_{b}$ phase formed by boardlike molecules.)

Yet another in-layer structure of the phase, with degenerate azimuthal orientation of the tilted monomers, is compatible with our x-ray scattering data. In this case, the para-axes of the monomers are randomly distributed on a cone, forming a "de Vries SmA" structure [86]. However, 


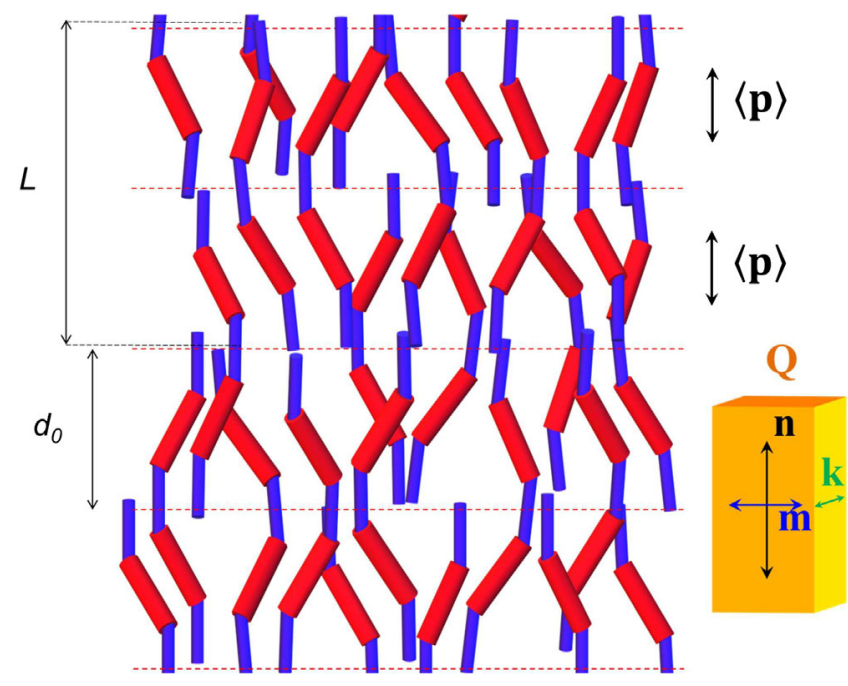

FIG. 25. Sketch of an $M_{X}$ phase with a $\operatorname{Sm} A_{b}\left(\operatorname{Sm} A_{b}\right)$ structure. As for the $\operatorname{Sm} A_{b}\left(\operatorname{SmC} C_{A}\right)$ structure presented in Fig. 3, the monomer units form smectic liquid layers with thickness $d_{0} \approx L / 2$. The dimers span again two adjacent layers and form an orthogonal intercalated smectic phase with biaxial orientational order parameter tensor $\mathbf{Q}$. However, in each layer, the average orientation of the monomer axis $\mathbf{p}$ is parallel to the layers normal, because the tilt of the monomers is doubly degenerated, which is quite unlikely due to a strong energetic penalty.

this highly symmetric phase is uniaxial and is, therefore, ruled out by our optical and electric data for the $M_{X}$ phase.

Our experimental data do not actually give any clear evidence about which of the $\operatorname{Sm} A_{b}\left(\operatorname{Sm} C_{A}\right)$ or $\operatorname{Sm} A_{b}\left(\operatorname{Sm} A_{b}\right)$ microscopic structures applies to the $M_{X}$ phase. Both structures should present similar elastic, optic, and dielectric properties. However, there are clear theoretical arguments in favor of the $\operatorname{Sm} A_{b}\left(\mathrm{SmC}_{A}\right)$ structure. Indeed, the relative stability of the two structures is defined by the balance between the energy and entropy terms, both positional and orientational, in the free energy. The condensation of the smectic order of the monomers indicates that the positional energy gain outweighs the positional entropy loss. The energy gain for parallel in-layer orientation of the monomers favors their uniform tilt within each layer, i.e., their SmC-like in-layer organization. Because the monomers are very anisotropic and may form mesophases when nondimerized, this energy term must be very large, of the order of several kT per monomer [87-89]. Note that, for $N_{\mathrm{TB}}$-forming compounds like BNA-76, the angle between two neighboring monomers tilted in opposite ways is approximately $60^{\circ}$, resulting in a prohibitively large energy cost. However, the orientational entropy term favors the $\operatorname{Sm} A_{b}$ organization of the monomers in the layer. Nevertheless, the tendency of the monomers to form uniaxial nematic phases strongly indicates that the orientational entropy term, which is even larger in the $N$ phase due to its higher orientational disorder, cannot balance the unfavorable interaction energy of the
$\operatorname{Sm} A_{b}\left(\operatorname{Sm} A_{b}\right)$ structure. Therefore, we conclude that the organization of the monomer layers in the $M_{X}$ phase is that shown in Fig. 3. Additional factors not discussed here, for example, the close packing of the monomers in the layer, should also favor the $\mathrm{SmC}_{A}$-like organization of the monomers.

[1] V. Fréedericksz and V. Zolina, Forces Causing the Orientation of an Anisotropic Liquid, Trans. Faraday Soc. 29, 919 (1933).

[2] M. Goscianski, L. Leger, and A. Mircea-Roussel, FieldInduced Transitions in Smectic A-Phases, J. Phys. (Paris), Lett. 36, L313 (1975).

[3] A. Rapini, Magnetic Instabilities of a Smectic-C, J. Phys. (Paris) 33, 237 (1972).

[4] D. R. Link, G. Natale, R. Shao, J. E. Maclennan, N. A. Clark, E. Korblova, and D. M. Walba, Spontaneous Formation of Macroscopic Chiral Domains in a Fluid Smectic Phase of Achiral Molecules, Science 278, 1924 (1997).

[5] T. Niori, T. Sekine, J. Watanabe, T. Furukawa, and H. Takezoe, Distinct Ferroelectric Smectic Liquid Crystals Consisting of Banana Shaped Achiral Molecules, J. Mater. Chem. 6, 1231 (1996).

[6] T. Sekine, T. Niori, J. Watanabe, T. Furukawa, S. W. Choi, and H. Takezoe, Spontaneous Helix Formation in Smectic Liquid Crystals Comprising Achiral Molecules, J. Mater. Chem. 7, 1307 (1997).

[7] J. Watanabe, T. Izumi, T. Niori, M. Zennyoji, Y. Takanishi, and H. Takezoe, Smectic Mesophase Properties of Dimeric Compounds. 2. Distinct Formation of Smectic Structures with Antiferroelectric Ordering and Frustration, Mol. Cryst. Liq. Cryst. 346, 77 (2000).

[8] C. T. Imrie, P. A. Henderson, and J. M. Seddon, Nonsymmetric Liquid Crystal Trimers. The First Example of a Triply-Intercalated Alternating Smectic C Phase, J. Mater. Chem. 14, 2486 (2004).

[9] G. S. Attard, R. W. Date, C. T. Imrie, G. R. Luckhurst, S. J. Roskilly, J. M. Seddon, and L. Taylor, Non-symmetric Dimeric Liquid Crystals-The Preparation and Properties of the alpha-(4-cyanobiphenyl-4'-yloxy)-omega-(4$n$-alkylanilinebenzylidene-4'-oxy) Alkanes, Liq. Cryst. 16, 529 (1994).

[10] R. J. Mandle and J. W. Goodby, A Twist-Bend Nematic to an Intercalated, Anticlinic, Biaxial Phase Transition in Liquid Crystal Bimesogens, Soft Matter 12, 1436 (2016).

[11] A. Knežević, I. Dokli, M. Sapunar, S. Šegota, U. Baumeister, and A. Lesac, Induced Smectic Phase in Binary Mixtures of Twist-Bend Nematogens, Beilstein J. Nanotechnol. 9, 1297 (2018).

[12] T. Hegmann, J. Kain, S. Diele, G. Pelzl, and C. Tschierske, Evidence for the Existence of the McMillan Phase in a Binary System of a Metallomesogen and 2,4,7-trinitrofluorenone, Angew. Chem., Int. Ed. 40, 887 (2001).

[13] W. Weissflog, S. Righter, E. Dietzmann, J. Risse, S. Diele, P. Schiller, and G. Pelzl, The Structure and Unusual Optical Textures of Smectic C-2 Phases of New Tail-to-Tail Twins, Cryst. Res. Technol. 32, 271 (1997). 
[14] H. R. Brand, P. E. Cladis, and H. Pleiner, Macroscopic Properties of Smectic C-G Liquid Crystals, Eur. Phys. J. B 6, 347 (1998).

[15] A. Eremin, S. Diele, G. Pelzl, H. Nadasi, W. Weissflog, J. Salfetnikova, and H. Kresse, Experimental Evidence for an Achiral Orthogonal Biaxial Smectic Phase without In-Plane Order Exhibiting Antiferroelectric Switching Behavior, Phys. Rev. E 64, 051707 (2001).

[16] R. B. Meyer, Structural Problems in Liquid Crystal Physics, in Molecular Fluids, edited by R. Balian and G. Weill (Gordon and Breach, New York, 1976), p. 273.

[17] I. Dozov, On the Spontaneous Symmetry Breaking in the Mesophases of Achiral Banana-Shaped Molecules, Europhys. Lett. 56, 247 (2001).

[18] V. P. Panov, M. Nagaraj, J. K. Vij, Y. P. Panarin, A. Kohlmeier, M. G. Tamba, R. A. Lewis, and G. H. Mehl, Spontaneous Periodic Deformations in Nonchiral PlanarAligned Bimesogens with a Nematic-Nematic Transition and a Negative Elastic Constant, Phys. Rev. Lett. 105, 167801 (2010).

[19] M. Cestari et al., Phase Behavior and Properties of the Liquid-Crystal Dimer 1"7"-bis(4-cyanobiphenyl-4'-yl) heptane: A Twist-Bend Nematic Liquid Crystal, Phys. Rev. E 84, 031704 (2011).

[20] V. P. Panov, R. Balachandran, M. Nagaraj, J. K. Vij, M. G. Tamba, A. Kohlmeier, and G. H. Mehl, Microsecond Linear Optical Response in the Unusual Nematic Phase of Achiral Bimesogens, Appl. Phys. Lett. 99, 261903 (2011).

[21] V. Borshch et al., Nematic Twist-Bend Phase with Nanoscale Modulation of Molecular Orientation, Nat. Commun. 4, 2635 (2013).

[22] D. Chen et al., Chiral Heliconical Ground State of Nanoscale Pitch in a Nematic Liquid Crystal of Achiral Molecular Dimers, Proc. Natl. Acad. Sci. U.S.A. 110, 15931 (2013).

[23] C. Meyer, G. R. Luckhurst, and I. Dozov, Flexoelectrically Driven Electroclinic Effect in the Twist-Bend Nematic Phase of Achiral Molecules with Bent Shapes, Phys. Rev. Lett. 111, 067801 (2013).

[24] S. M. Shamid, S. Dhakal, and J. V. Selinger, Statistical Mechanics of Bend Flexoelectricity and the Twist-Bend Phase in Bent-Core Liquid Crystals, Phys. Rev. E 87, 052503 (2013).

[25] C. Zhu, M. R. Tuchband, A. Young, M. Shuai, A. Scarbrough, D. M. Walba, J. E. Maclennan, C. Wang, A. Hexemer, and N. A. Clark, Resonant Carbon K-Edge Soft $X$-Ray Scattering from Lattice-Free Heliconical Molecular Ordering: Soft Dilative Elasticity of the Twist-Bend Liquid Crystal Phase, Phys. Rev. Lett. 116, 147803 (2016).

[26] A. Knežević, M. Sapunar, A. Buljan, I. Dokli, Z. Hameršak, D. Kontrec, and A. Lesac, Fine-Tuning the Effect of pi-pi Interactions on the Stability of the N-TB Phase, Soft Matter 14, 8466 (2018).

[27] N. Chaturvedi and R. D. Kamien, Mechanisms to SplayBend Nematic Phases, Phys. Rev. E 100, 022704 (2019).

[28] N. Vaupotič, M. Čepič, M. A. Osipov, and E. Gorecka, Flexoelectricity in Chiral Nematic Liquid Crystals as a Driving Mechanism for the Twist-Bend and Splay-Bend Modulated Phases, Phys. Rev. E 89, 030501(R) (2014).
[29] G. Pajak, L. Longa, and A. Chrzanowska, Nematic TwistBend Phase in an External Field, Proc. Natl. Acad. Sci. U.S.A. 115, E10303 (2018).

[30] G. Barbero and I. Lelidis, Fourth-Order Nematic Elasticity and Modulated Nematic Phases: A Poor Man's Approach, Liq. Cryst. 46, 535 (2019).

[31] C. Meyer, C. Blanc, G. R. Luckhurst, P. Davidson, and I. Dozov, Biaxiality-Driven Twist-Bend to Splay-Bend Nematic Phase Transition Induced by an Electric Field, Sci. Adv. 6, eabb8212 (2020).

[32] J. Watanabe, H. Komura, and T. Niori, Thermotropic Liquid-Crystals of Polyesters Having a Mesogenic 4,4bibenzoate Unit-Smectic Mesophase Properties and Structures in Dimeric Model Compounds, Liq. Cryst. 13, 455 (1993).

[33] P. J. Le Masurier and G. R. Luckhurst, Structural Studies of the Intercalated Smectic C Phases Formed by the Non-symmetric alpha-(4-cyanobiphenyl-4'-yloxy)-omega(4-alkylaniline-benzylidene-4 '-oxy) Alkane Dimers Using EPR Spectroscopy, J. Chem. Soc., Faraday Trans. 94, 1593 (1998).

[34] W. Weissflog, C. Lischka, S. Diele, I. Wirth, and G. Pelzl, The Inverse Phase Sequence SmA-SmC in Symmetric Dimeric Liquid Crystals, Liq. Cryst. 27, 43 (2000).

[35] D. Pociecha, D. Kardas, E. Gorecka, J. Szydlowska, J. Mieczkowski, and D. Guillon, Modulated and Intercalated Smectic Phases Formed by Dimeric Molecules, J. Mater. Chem. 13, 34 (2003).

[36] P. A. Henderson and C. T. Imrie, Non-symmetric Liquid Crystal Trimers, Liq. Cryst. 32, 673 (2005).

[37] C. T. Imrie, Non-symmetric Liquid Crystal Dimers: How to Make Molecules Intercalate, Liq. Cryst. 33, 1449 (2006).

[38] C. T. Imrie and P. A. Henderson, Liquid Crystal Dimers and Higher Oligomers: Between Monomers and Polymers, Chem. Soc. Rev. 36, 2096 (2007).

[39] M. Šepelj, A. Lesac, U. Baumeister, S. Diele, H. L. Nguyen, and D. W. Bruce, Intercalated Liquid-Crystalline Phases Formed by Symmetric Dimers with an alpha,omegadiiminoalkylene Spacer, J. Mater. Chem. 17, 1154 (2007).

[40] T. Donaldson, H. Staesche, Z. B. Lu, P. A. Henderson, M. F. Achard, and C. T. Imrie, Symmetric and Non-symmetric Chiral Liquid Crystal Dimers, Liq. Cryst. 37, 1097 (2010).

[41] T. Ivšić, U. Baumeister, I. Dokli, A. Mikleušević, and A. Lesac, Sensitivity of the N-TB Phase Formation to the Molecular Structure of Imino-Linked Dimers, Liq. Cryst. 44, 93 (2017).

[42] E. J. Davis and J. W. Goodby, Classification of Liquid Crystals According to Symmetry, in Handbook of Liquid Crystals, edited by J. W. G. Goodby et al. (Wiley VCH, Weinheim, 2014), p. 27.

[43] HyperChem(TM) Professional 7.51 (Hypercube, Inc., 1115 NW 4th Street, Gainesville, Florida 32601, USA, n.d.).

[44] R. J. Mandle and J. W. Goodby, Intercalated SoftCrystalline Mesophase Exhibited by an Unsymmetrical Twist-Bend Nematogen, CrystEngComm 18, 8794 (2016).

[45] R. J. Mandle, S. J. Cowling, and J. W. Goodby, Combined Microscopy, Calorimetry and X-Ray Scattering Study of Fluorinated Dimesogens, Sci. Rep. 7, 13323 (2017). 
[46] T. Donaldson, P. A. Henderson, M. F. Achard, and C. T. Imrie, Chiral Liquid Crystal Tetramers, J. Mater. Chem. 21, 10935 (2011).

[47] C. T. Imrie and P. A. Henderson, Liquid Crystal Dimers and Oligomers, Curr. Opin. Colloid Interface Sci. 7, 298 (2002).

[48] C. T. Imrie, P. A. Henderson, and G. Y. Yeap, Liquid Crystal Oligomers: Going beyond Dimers, Liq. Cryst. 36, 755 (2009).

[49] Y. I. Suzuki, T. Isozaki, S. Hashimoto, T. Kusumoto, T. Hiyama, Y. Takanishi, H. Takezoe, and A. Fukuda, Stability of the Antiferroelectric Phase in Dimeric Liquid Crystals Having Two Chiral Centres with CF3 or CH3 Groups; Evaluation of Conformational and Electric Interactions, J. Mater. Chem. 6, 753 (1996).

[50] Y. Ouchi, Y. Yoshioka, H. Ishii, K. Seki, M. Kitamura, R. Noyori, Y. Takanishi, and I. Nishiyama, Effect of the Terminal Branching Structure of Some Liquid-Crystalline Biphenyl Carboxylates on the Stability of the Antiferroelectric Phase, J. Mater. Chem. 5, 2297 (1995).

[51] P. G. de Gennes and J. Prost, The Physics of Liquid Crystals (Clarendon, Oxford, 1994).

[52] See Supplemental Material at http://link.aps.org/ supplemental/10.1103/PhysRevX.11.031012 for video of the coarsening process of the twin Fréedericksz domains.

[53] I. Haller, Thermodynamic and Static Properties of Liquid Crystals, Prog. Solid State Chem. 10, 103 (1975).

[54] C. Meyer, G. R. Luckhurst, and I. Dozov, The Temperature Dependence of the Heliconical Tilt Angle in the Twist-Bend Nematic Phase of the Odd Dimer CB7CB, J. Mater. Chem. C 3, 318 (2015).

[55] G. Cukrov, Y. M. Golestani, J. Xiang, Y. A. Nastishin, Z. Ahmed, C. Welch, G. H. Mehl, and O. D. Lavrentovich, Comparative Analysis of Anisotropic Material Properties of Uniaxial Nematics Formed by Flexible Dimers and Rod-like Monomers, Liq. Cryst. 44, 219 (2017).

[56] V.P. Panov, J. K. Vij, and G. H. Mehl, Twist-Bend Nematic Phase in Cyanobiphenyls and Difluoroterphenyls Bimesogens, Liq. Cryst. 44, 147 (2017).

[57] D. Pociecha, C. A. Crawford, D. A. Paterson, J. M. D. Storey, C. T. Imrie, N. Vaupotič, and E. Gorecka, Critical Behavior of the Optical Birefringence at the Nematic to Twist-Bend Nematic Phase Transition, Phys. Rev. E 98, 052706 (2018).

[58] A. Bogi and S. Faetti, Elastic, Dielectric and Optical Constants of 4'-pentyl-4-cyanobiphenyl, Liq. Cryst. 28, 729 (2001).

[59] S. W. Morris, P. Palffy-Muhoray, and D. A. Balzarini, Measurements of the Bend and Splay Elastic-Constants of Octylcyanobiphenyl, Mol. Cryst. Liq. Cryst. 139, 263 (1986).

[60] T. Uchida and Y. Takahashi, New Method to Determine Elastic-Constants of Nematic Liquid-Crystal from $C-V$ Curve, Mol. Cryst. Liq. Cryst. 72, 133 (1981).

[61] F. C. Frank, I. Liquid Crystals. On the Theory of Liquid Crystals, Faraday Discuss. 25, 19 (1958).

[62] E. Govers and G. Vertogen, Elastic Continuum Theory of Biaxial Nematics, Phys. Rev. A 30, 1998 (1984).

[63] A. Saupe, Elastic and Flow Properties of Biaxial Nematics, J. Chem. Phys. 75, 5118 (1981).
[64] H. J. Deuling, Deformation of Nematic Liquid-Crystals in an Electric-Field, Mol. Cryst. Liq. Cryst. 19, 123 (1972).

[65] H. Gruler, T. J. Scheffer, and G. Meier, Elastic-Constants of Nematic Liquid-Crystals. 1. Theory of Normal Deformation, Z. Naturforsch. A 27, 966 (1972).

[66] Y. A. Nastishin, R. D. Polak, S. V. Shiyanovskii, V. H. Bodnar, and O. D. Lavrentovich, Nematic Polar Anchoring Strength Measured by Electric Field Techniques, J. Appl. Phys. 86, 4199 (1999).

[67] K. Adlem, M. Čopič, G. R. Luckhurst, A. Mertelj, O. Parri, R. M. Richardson, B. D. Snow, B. A. Timimi, R. P. Tuffin, and D. Wilkes, Chemically Induced Twist-Bend Nematic Liquid Crystals, Liquid Crystal Dimers, and Negative Elastic Constants, Phys. Rev. E 88, 022503 (2013).

[68] C.-J. Yun, M. R. Vengatesan, J. K. Vij, and J.-K. Song, Hierarchical Elasticity of Bimesogenic Liquid Crystals with Twist-Bend Nematic Phase, Appl. Phys. Lett. 106, 173102 (2015).

[69] E. Govers and G. Vertogen, Fluid-Dynamics of Biaxial Nematics, Physica (Amsterdam) 133A, 337 (1985).

[70] M. A. Osipov and A. M. Sonnet, Order Parameter Dependence of the Viscosity Coefficients of a Biaxial Nematic Liquid Crystal, Eur. Phys. J. E 34, 109 (2011).

[71] M. Ricci, R. Berardi, and C. Zannoni, On the Field-Induced Switching of Molecular Organization in a Biaxial Nematic Cell and Its Relaxation, J. Chem. Phys. 143, 084705 (2015).

[72] P. Oswald and P. Pieranski, Nematic and Cholesteric Liquid Crystals: Concepts and Physical Properties Illustrated by Experiments, 1st ed. (CRC Press, Boca Raton, FL, 2005).

[73] C. Meyer and I. Dozov, Local Distortion Energy and Coarse-Grained Elasticity of the Twist-Bend Nematic Phase, Soft Matter 12, 574 (2016).

[74] A. Jákli, Fast Switching Electro-Optical Devices Using Banana-Shaped Liquid Crystals, U.S. Patent 7,782,438, 2010.

[75] M. Nagaraj, Y. P. Panarin, U. Manna, J. K. Vij, C. Keith, and C. Tschierske, Electric Field Induced Biaxiality and the Electro-Optic Effect in a Bent-Core Nematic Liquid Crystal, Appl. Phys. Lett. 96, 011106 (2010).

[76] M. Čopič and A. Mertelj, Q-Tensor Model of Twist-Bend and Splay Nematic Phases, Phys. Rev. E 101, 022704 (2020).

[77] R. J. Mandle and A. Mertelj, Orientational Order in the Splay Nematic Ground State, Phys. Chem. Chem. Phys. 21, 18769 (2019).

[78] A. Mertelj, L. Cmok, N. Sebastian, R. J. Mandle, R. R. Parker, A. C. Whitwood, J. W. Goodby, and M. Čopič, Splay Nematic Phase, Phys. Rev. X 8, 041025 (2018).

[79] R. Walker, D. Pociecha, J. M. D. Storey, E. Gorecka, and C. T. Imrie, Remarkable Smectic Phase Behaviour in OddMembered Liquid Crystal Dimers: The CT6O.m Series, J. Mater. Chem. C 9, 5167 (2021).

[80] P. Davidson, P. Keller, and A. M. Levelut, MolecularOrganization in Side-Chain Liquid-Crystalline Polymers, J. Phys. (Paris) 46, 939 (1985).

[81] J. Ilavsky, Nika: Software for Two-Dimensional Data Reduction, J. Appl. Crystallogr. 45, 324 (2012). 
[82] M. Born and E. Wolf, Principles of Optics: Electromagnetic Theory of Propagation, Interference and Diffraction of Light (Cambridge University Press, Cambridge, England, 2000).

[83] C. Maze and D. Johnson, Determination of Nematic LiquidCrystal Elastic and Dielectric-Constants from Birefringence Experiments, Mol. Cryst. Liq. Cryst. 33, 213 (1976).

[84] S. Joly, K. Antonova, P. Martinot-Lagarde, and I. Dozov, Zenithal Gliding of the Easy Axis of a Nematic Liquid Crystal, Phys. Rev. E 70, 050701(R) (2004).

[85] L. M. Blinov, Structure and Properties of Liquid Crystals (Springer, Dordrecht, 2011).
[86] A. de Vries, Experimental-Evidence Concerning 2 Different Kinds of Smectic-C to Smectic-A Transitions, Mol. Cryst. Liq. Cryst. 41, 27 (1977).

[87] W. Maier and A. Saupe, Eine Einfache Molekulare Theorie des Nematischen Kristallinflussigen Zustandes, Z. Naturforsch. 13 a, 564 (1958).

[88] W. Maier and A. Saupe, Eine Einfache Molekular-Statistische Theorie der Nematischen Kristallinflussigen Phase.1, Z. Naturforsch. 14 a, 882 (1959).

[89] W. Maier and A. Saupe, Eine Einfache Molekular-Statistische Theorie der Nematischen Kristallinflussigen Phase. 2, Z. Naturforsch. 15 a, 287 (1960). 